\title{
Distributed Energy Spectral Efficiency Optimization for Partial/Full Interference Alignment in Multi-user Multi-relay Multi-cell MIMO Systems
}

\author{
Kent Tsz Kan Cheung, Shaoshi Yang, Member, IEEE, and Lajos Hanzo, Fellow, IEEE
}

\begin{abstract}
The energy spectral efficiency maximization (ESEM) problem of a multi-user, multi-relay, multi-cell system is considered, where all the network nodes are equipped with multi-antenna transceivers. To deal with the potentially excessive interference originating from a plethora of geographically distributed transmission sources, a pair of transmission protocols based on interference alignment (IA) are conceived. The first, termed the full-IA, avoids all intra-cell interference (ICI) and other-cell interference by finding the perfect interference-nulling receive beamforming matrices (RxBFMs). The second protocol, termed partial-IA, only attempts to null the ICI. Employing the RxBFMs computed by either of these protocols mathematically decomposes the channel into a multiplicity of non-interfering multiple-input-single-output channels, which we term as spatial multiplexing components (SMCs). The problem of finding the optimal SMCs as well as their power control variables for the ESEM problem considered is formally defined and converted into a convex optimization form with carefully selected variable relaxations and transformations. Thus, the optimal SMCs and power control variables can be distributively computed using both the classic dual decomposition and subgradient methods. Our results indicate that indeed, the ESEM algorithm performs better than the baseline equal power allocation algorithm in terms of its ESE. Furthermore, surprisingly the partial-IA outperforms the full-IA in all cases considered, which is because the partial-IA is less restrictive in terms of the number of available transmit dimensions at the transmitters. Given the typical cell sizes considered in this paper, the path-loss sufficiently attenuates the majority of the interference, and thus the full-IA over-compensates, when trying to avoid all possible sources of interference.
\end{abstract}

Index Terms-Distributed optimization, energy efficiency, fractional programming, green communications, interference alignment (IA), multiple-input-multiple-output (MIMO).

Manuscript received July 03, 2015; accepted October 01, 2015. Date of publication October 26, 2015; date of current version nulldate. The associate editor coordinating the review of this manuscript and approving it for publication was Dr. Marius Pesavento. This work was supported by the Industrial Companies who are Members of the Mobile VCE, with additional support by the U.K. Government's Engineering \& Physical Sciences Research Council (EPSRC). The financial support of the Research Councils U.K. (RCUK) under the IndiaU.K. Advanced Technology Center (IU-ATC), of the E.U. under the auspices of the Concerto project, and of the European Research Council's Senior Research Fellow Grant is also gratefully acknowledged.

The authors are with the School of Electronics and Computer Science, University of Southampton, Southampton, SO17 1BJ, U.K. (e-mail: ktkc106@ecs. soton.ac.uk; shaoshi.yang@ecs.soton.ac.uk; 1h@ecs.soton.ac.uk).

Color versions of one or more of the figures in this paper are available online at http://ieeexplore.ieee.org.

Digital Object Identifier 10.1109/TSP.2015.2488579

\section{INTRODUCTION}

\section{$\mathbf{F}$} UTURE wireless cellular networks are required to satisfy ever-increasing area spectral efficiency (ASE) demands in the context of densely packed heterogeneous cells, where both relay nodes (RNs) and small-cells [1], [2] are employed. However, these changes will result in severe co-channel interference (CCI), since future networks will aim for fully exploiting the precious wireless spectrum by relying on a unity frequency reuse factor [3]. Furthermore, owing to the growing energy costs, a system's energy efficiency is becoming a major concern [4]. Against this backdrop, in this paper we aim for maximizing the energy spectral efficiency (ESE) of the downlink (DL) of a decode-and-forward (DF) [5] relay-aided multiple-input-multiple-output orthogonal frequency division multiple access (MIMO-OFDMA) multi-cell network that employs the technique of interference alignment (IA).

IA was first introduced in [6]-[8], and it was further popularized in [9], [10]. In [10], Cadambe et al. described the main concept of IA and established the attainable degrees of freedom (DoF), when employing IA for completely avoiding interference in a network supporting $K$ user-pairs. The principle of IA is that, instead of dividing the wireless resources amongst all users (often termed as orthogonalization), each user aligns his/her transmissions into a predetermined subspace, referred to as the interference subspace, at all the other receivers, so that the remaining subspace at all receivers becomes free of interference. Thus, the attainable DoFs in a system supporting $K$ user-pairs is $K / 2$ when employing IA, instead of $1 / K$ obtained through orthogonalization [10]. This becomes highly favorable, as $K$ increases.

Hence, IA has been advocated as a viable technique of managing the uplink (UL) CCI of multi-cell networks [11], [12]. Explicitly, IA is suitable for the UL, since the number of receive antennas (RAs) at the basestation (BS) is typically higher than the number of transmit antennas (TAs) at each user equipment (UE). Thus, the potentially higher number of signal dimensions available at the receiver can be exploited for aligning the CCI into a predetermined interference subspace, so that the $\mathrm{BS}$ can receive the transmissions of its own UEs without CCI. However, this is not feasible in the DL, since each UE has access to a low number of receive dimensions. This challenge was successfully tackled by the DL transmission scheme of [13], which relies on specifically designing transmit precoding (TP) matrices for reducing the number of transmit dimensions at the 
BSs, thus facilitating DL IA at the UEs. In contrast to other IA techniques, such as [14]-[18], the technique presented in [13] does not require cooperation among the BSs for exchanging channel state information (CSI), and IA is accomplished distributively. Furthermore, this technique facilitates IA in systems relying on arbitrary antenna configurations with the aid of frequency- or time-extension, which is capable of substantially expanding the total number of transmit and receive dimensions in a multicarrier system such as OFDMA. In [19], the technique of [13] was generalized to an arbitrary number of BSs and UEs, where each of them is equipped with an arbitrary number of antennas as well. Furthermore, the authors of [19] employed the semi-orthogonal user selection scheme of Yoo et al. [20] for maximizing the achievable spectral efficiency (SE). However, relaying was not considered in [19] and each UE was limited to receiving a single spatial stream.

In this paper, we aim for maximizing the system's attainable ESE, defined as a counterpart of ASE [21], where the latter has the units of (bits/sec/Hz/km ${ }^{2}$ ), while the former is measured in (bits/sec/Hz/Joule). This ESE metric has also been utilized in [22]-[27]. The authors of [22] considered ESE maximization (ESEM) of both the UL and the DL of a cellular network, while providing both the optimal solution method and a lower-complexity heuristic method. However, the effects of interference were not quantified in the system model of [22], since only a single cell was considered. Additionally, no relaying was employed. In [23], ESEM was performed in a multi-cell setting, where the CCI was eliminated with the aid of BS cooperation [28] and zero-forcing beamforming (ZFBF). However, the authors of [23] have not considered the benefits of multiple antenna aided nodes or relaying. As a further advance, the energy-efficiency of a relay aided system was considered in [24], where the objective function (OF) of the optimization problem considered was formulated as the weighted sum of both the SE and the energy dissipated. Nevertheless, these two metrics must be appropriately weighted, which is still an open challenge. Thus, the ESE metric was not formally optimized.

In fact, the maximization of the ESE metric is typically formulated as a fractional (in this case, quasi-concave) programming problem [29]-[31], which relies on the classic solution methods of the bisection search [31], and on Dinkelbach's method [29], as employed in [23], [25], [26]. However, the bisection search requires solving multiple convex feasibility problems, while Dinkelbach's method requires solving multiple concave subtractive optimization problems. The total number of algorithmic iterations may become prohibitive in both cases. Hence, we opt for employing a beneficial method based on the Charnes-Cooper variable transformation [30], [32], allowing us to solve the ESEM problem by solving a single concave optimization problem and to demonstrate its benefits to the wireless communications community.

Let us now elaborate further by classifying the $\mathrm{CCI}$ as intracell interference (ICI) and other-cell interference (OCI). In the DL considered, the former describes the interference that a RN or UE may receive from the BS within its own cell, where multiple concurrent transmissions are also intended for other RNs or UEs, while the latter describes the interference originating from sources located in other cells.
We now provide a concise list of the contributions presented in this paper.

- We evaluate the ESEM of IA employed in a realistic MIMO-OFDMA system involving multiple cells, multiple relays and multiple users. Although ESEM has been studied intensely in recent years [22]-[24], these contributions typically consider a single cell providing coverage without the assistance of relaying, or do not exploit the benefits of multi-antenna aided transceivers. Additionally, although IA was employed recently in [14], [33]-[36], these contributions focus on user-pair networks rather than on multi-user cellular networks, and the associated challenges of implementing IA require further research in the latter scenario. More importantly, previous contributions typically aim for investigating its SE benefits, while the achievable ESE of using IA-based protocols has not been explored at all. Green communications has become increasingly important, but the quantitative benefits of IA have not been documented in the context of energy-efficient communications. Therefore, in this contribution we seek to deepen the research community's understanding of IA from an ESE perspective. Furthermore, a more realistic multi-cell MIMO-OFDMA relay-aided network is considered in this treatise, where multiple users are supported by each BS and multiple relays. Therefore, the system model considered inevitably becomes challenging. As a beneficial result, the protocols and solutions provided in this paper can be more readily applied to real network scenarios, when compared to the existing IA literature, which focuses only on the $K$-user interference network. In contrast to our previous contributions [25]-[27], this treatise investigates a multi-antenna aided multi-cell system. Although a multi-antenna assisted system was also studied in our previous contribution [27], only a single macrocell was considered and no IA was employed for avoiding the ICI imposed by both the simultaneously transmitting BS and RNs.

- We provide a sophisticated generalization of the IA protocol considered in [13]. Explicitly, in contrast to [13], the proposed IA protocol accounts for three cells, for an arbitrary number of users in each cell, for an arbitrary antenna configuration, and for simultaneous direct as well as relay-aided transmissions. This is accomplished through the careful design of precoding-, transmit- and receive-beamforming matrices in order to ensure that IA is achieved. In particular, the number of guaranteed spatial dimensions available at the BSs, RNs and UEs must be judiciously chosen. Furthermore, we conceive two transmission protocols in this work, which may be implemented distributively at each BS. The first protocol is termed as full-IA, which invokes IA for avoiding the interference arriving from all transmitters. This is the intuitive choice, as advocated by the existing literature [11], [13], [19] highlighting its benefits in terms of achieving the optimal DoF. Note that the full-IA scheme employed in [13], but was designed for a simpler system model having no relays. The second protocol proposed is unlike that of [13] and it is termed as partial-IA, which only aims 
for avoiding the ICI using IA, while ignoring the effect of OCI when making scheduling decisions. The partial-IA protocol therefore reduces the computational burden of having to estimate the DL CSI of the other-cell channel matrices at the receivers, albeit this might be expected to reduce the system's performance due to neglecting the OCI. We compared the performance of these two protocols and found that, as a surprise, the reduced-complexity partial-IA protocol is potentially capable of achieving a higher ESE than the full-IA protocol. Explicitly, this is because more simultaneous transmissions may be scheduled due to the partial-IA protocol's relaxed constraint on the number of transmit dimensions available. Furthermore, in contrast to the protocol proposed in [19], ours is a two-phase protocol, which is specifically designed for relay-aided networks and does not limit the number of spatial streams available to each UE.

- Employing the beamforming matrices calculated from either the full-IA or partial-IA protocols results in a list of spatial multiplexing components (SMCs) ${ }^{1}$, which correspond to the specific data streams that the BSs can choose to support. Finding the optimal SMCs as well as the optimal power control variables associated with these optimal SMCs is formally defined as a network-wide optimization problem. Unlike in our previous work [25]-[27], we decompose the network-wide multi-cell optimization problem in order to formulate a subproblem for each BS using the technique of primal decomposition [37], thus eliminating the need for the high-overhead backhaul-aided message passing amongst the BSs. Each of these subproblems is then converted into a convex form with the aid of various variable relaxations and transformations, which can then be optimally and distributively solved using the dual decomposition and subgradient methods of [37].

The organization of this paper is as follows. We introduce our system model in Section II and describe the proposed transmission protocols in Section III. Subsequently, the ESEM problem considered is formulated in Section IV, where the solution method is developed as well. Our numerical results along with our further discussions are presented in Section V. Finally, our conclusions are given in Section VI along with our future research ideas.

\section{SYSTEM MODEL}

In this work, a multi-cell DL MIMO-OFDMA network, relying on a radical unity frequency reuse factor is considered. The ubiquitous OFDMA technique is employed for avoiding the severe frequency-selective fading encountered in wideband communication systems. Additionally, OFDMA allows for transmission symbol extensions in the frequency-domain [13], which are required by the proposed IA-based transmission protocol described in Section III.

As depicted in Fig. 1, each macrocell is divided into three sectors, and it is assumed that the employment of directional antennas and the non-line-of-sight (NLOS) path-loss attenuates the interference power, with the exception of the OCI received from the first tier of interfering cells and the ICI from the serving

${ }^{1}$ These SMCs are detailed further in Section III.
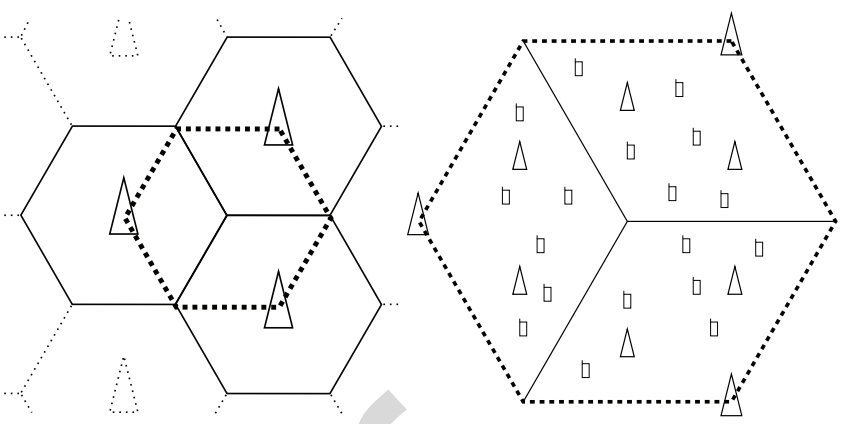

Fig. 1. A multi-cell system is depicted on the left. Each cell is divided into three sectors, and one sector from each of the three neighboring cells is individually highlighted. This highlighted region is termed an OCI region. Through the use of directional antennas, it is assumed that the main source of OCI is caused when the neighboring BSs simultaneously transmit to a receiver located in their associated OCI region. On the right is a close-up view of the OCI region, with three BSs at the vertices of its perimeter. Furthermore, each sector is supported by two RNs and provides coverage for six UEs in this example.

BS and RNs of each macrocell. Therefore, we may focus our attention on the central region seen at the left of Fig. 1, which we term as an OCI region. Thus, each DL transmission within an OCI region is subjected to OCI from two macrocells. Furthermore, each $120^{\circ}$-sector of Fig. 1 is supported by $M$ RNs, which are located at a fixed distance from the associated BS and evenly spaced within the sector, as seen on the right of Fig. 1. The ratio of the BS-RN distance to the cell radius is denoted by $D_{r}$. Additionally, $K$ UEs are uniformly distributed within each $120^{\circ}$-sector. The system has access to $L$ OFDMA subcarrier blocks, each characterized by a wireless bandwidth of $W$ Hertz. The BSs, DF RNs, and UEs are respectively equipped with $N_{B}, N_{R}$ and $N_{U}$ antennas. It is assumed that all BSs and RNs are synchronized when they transmit to the UEs, and that the transmitters employ complex-valued symbol constellations to convey their data.

For each subcarrier block $l \in\{1, \ldots, L\}$, the complex-valued channel matrix associated with the wireless link spanning from the BS of macrocell $n^{\prime} \in\{1,2,3\}$ to RN $m \in\{1, \ldots, M\}$ belonging to macrocell $n \in\{1,2,3\}$ is denoted by $\mathbf{H}_{n, m}^{B R, l, n^{\prime}} \in \mathbb{C}^{N_{R} \times N_{B}}$. The channel matrix associated with the link spanning from the BS of macrocell $n^{\prime}$ to UE $k \in\{1, \ldots, K\}$ belonging to macrocell $n$ on subcarrier block $l$ is denoted by $\mathbf{H}_{n, k}^{B U, l, n^{\prime}} \in \mathbb{C}^{N_{U} \times N_{B}}$. Furthermore, the channel matrix associated with the link between $\mathrm{RN} m^{\prime}$ belonging to macrocell $n^{\prime}$ and UE $k$ belonging to macrocell $n$ on subcarrier block $l$ is denoted by $\mathbf{H}_{n, k}^{R U, l, n^{\prime}, m^{\prime}} \in \mathbb{C}^{N_{U} \times N_{R}}$. All channel matrices are assumed to have a full rank, as is often the case for wireless DL channels. For simplicity, the channel matrices associated with the same transceivers are combined across subcarrier blocks to give the block-diagonal channel matrices $\mathbf{H}_{n, m}^{B R, n^{\prime}} \in \mathbb{C}^{L N_{R} \times L N_{B}}, \mathbf{H}_{n, k}^{B U, n^{\prime}} \in \mathbb{C}^{L N_{U} \times L N_{B}}$ and $\mathbf{H}_{n, k}^{R U, n^{\prime}, m^{\prime}} \in \mathbb{C}^{L N_{U} \times L N_{R}}$, respectively. For example, we have

$$
\mathbf{H}_{n, m}^{B R, n^{\prime}}:=\left[\begin{array}{ccc}
\mathbf{H}_{n, m}^{B R, 1, n^{\prime}} & \mathbf{0} & \mathbf{0} \\
\mathbf{0} & \ddots & \mathbf{0} \\
\mathbf{0} & \mathbf{0} & \mathbf{H}_{n, m}^{B R, L, n^{\prime}}
\end{array}\right] .
$$

\footnotetext{
${ }^{2}$ Superscript indices refer to the transmitter, while subscript indices refer to the receiver. Additionally, a prime symbol' refers to a potentially interfering transmission source.
} 


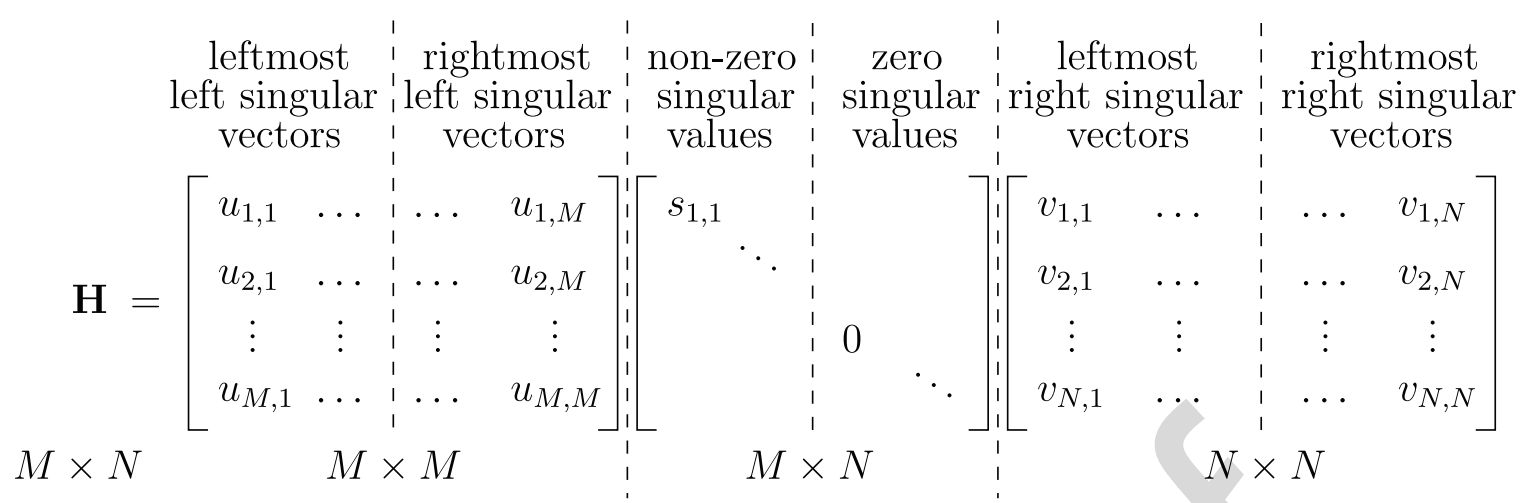

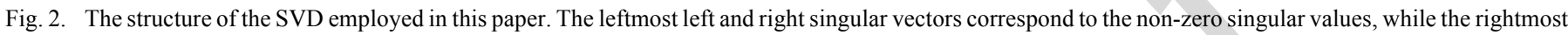
left and right singular vectors correspond to the zero singular values. Therefore, the rightmost left singular vectors span the left nullspace of $\mathbf{H}$.

The channel matrices account for both the small-scale frequency-flat Rayleigh fading, as well as the large-scale path-loss between the corresponding transceivers. In this system model, the transceivers are either stationary or moving sufficiently slowly for ensuring that the channel matrices can be considered time-invariant for the duration of a scheduled transmission period. However, the channel matrices may evolve between each transmission period. Furthermore, it is assumed that the transceivers' antennas are spaced sufficiently far apart for ensuring that the associated transmissions experience i.i.d. small-scale fading, whose coefficients are drawn from complex i.i.d. normal distributions having a zero mean and a unit variance. The system uses time-division duplexing (TDD) and hence the associated channel reciprocity may be exploited for predicting the CSI of the slowly varying DL channels from the received UL signal. Furthermore, by assuming the availability of low-rate error-free wireless backhaul channels, the CSI associated with the wireless intra-cell RN-UE links may be fed back to the particular BS in control, so that it may make the necessary scheduling decisions.

Additionally, each receiver suffers from complex-valued additive white Gaussian noise (AWGN) having a power spectral density of $N_{0}$. Due to both regulatory and safety concerns, the maximum instantaneous transmission power of each BS and each RN is limited, which is denoted by $P_{\max }^{B}$ and $P_{\max }^{R}$, respectively. We stipulate the idealized simplifying assumption that OFDMA modulation and demodulation is performed perfectly for all the information symbols.

\section{TRAnSmission Protocol Design}

Each BS may convey information to the UEs by either using a direct BS-UE link, or by utilizing a RN to create a two-hop BS-RN-UE link, which requires two transmission phases. Thus, each transmission period is split into two halves. Due to the simultaneous transmissions from multiple sources, both the ICI and OCI in the network are likely to be detrimental to the achievable ESE. In order to avoid both types of interference, the technique of IA is employed, which requires the careful design of the transmit beamforming matrices (TxBFMs) of both the BSs and of the RNs, as well as the receive beamforming matrices (RxBFMs) of both the RNs and of the UEs. As relaying links may be utilized in this system, the design of these matrices is different for the two transmission phases. Hence they are described separately in the following. Additionally, the full-IA and partial-IA protocols will be described side-by-side. To elaborate a little further, the full-IA protocol aims for completely avoiding both the ICI and OCI in both the first and second transmission phases, while the partial-IA protocol only aims for avoiding the ICI in both transmission phases, thus dispensing with estimating the OCI channel matrices at each receiver.

Furthermore, the proposed schemes crucially rely on the singular value decomposition (SVD), where the columns of the left and right singular matrices are composed of the left and right singular vectors of the associated matrix. These left and right singular vectors may be further partitioned into the leftmost and rightmost parts, which correspond to the non-zero and zero singular values, respectively. This structure is illustrated in detail in Fig. 2.

\section{A. Beamforming Design for the First Phase}

In the first phase, only the BSs are transmitting to both the RNs and the UEs. Therefore, the only source of interference is constituted by the neighboring BSs associated with the same OCI region. This interference may be avoided by carefully designing the TxBFMs at the BSs, as well as the RxBFMs at the RNs and the UEs in a distributive manner. Initially, a TP matrix, denoted by $\mathbf{A}^{B, n, T_{1}} \in \mathbb{C}^{L N_{B} \times S^{B, T_{1}}}$, is randomly generated for each BS $n$. Furthermore, $S^{B, T_{1}}$ is the number of symbols transmitted by each BS during the first phase, which is accurately defined in Sections III.A1 and and III.A2. The matrix $\mathbf{A}^{B, n, T_{1}}$ has a full column rank and its entries are complex-valued. These TP matrices are invoked for reducing the number of transmit dimensions available at each BS from $L N_{B}$ to $S^{B, T_{1}}$, thus facilitating IA at the receivers. Furthermore, the columns of these TP matrices are normalized so that the power assigned to each transmission remains unaffected. By employing these TP matrices, the precoded channel matrices of the first phase are given by

$$
\widetilde{\mathbf{H}}_{n, m}^{B R, n^{\prime}, T_{1}}:=\mathbf{H}_{n, m}^{B R, n^{\prime}} \mathbf{A}^{B, n^{\prime}, T_{1}} \in \mathbb{C}^{L N_{R} \times S^{B, T_{1}}}
$$

and

$$
\widetilde{\mathbf{H}}_{n, k}^{B U, n^{\prime}, T_{1}}:=\mathbf{H}_{n, k}^{B U, n^{\prime}} \mathbf{A}^{B, n^{\prime}, T_{1}} \in \mathbb{C}^{L N_{U} \times S^{B, T_{1}}},
$$

respectively for the BS-RN and BS-UE links. 
We now define $S^{R}$ and $S^{U}$ as the minimum number of receive dimensions at each RN and each UE, respectively, and both the parameters are chosen by the network operator. Furthermore, only the specific values of $S^{R}$ and $S^{U}$ along with the number of antennas at each network node and the number of available subcarrier blocks affect the feasibility of IA, while $M$ and $K$ have no effect.

1) Full-IA Receiver Design: In order to completely avoid the interference arriving from the neighboring BSs during the first phase, it is necessary for the precoded OCI channel matrices given by (2) and (3), to have intersecting left nullspaces. Firstly, the precoded OCI channel matrices may be concatenated for forming the interference matrices, for example

$$
\widehat{\mathbf{H}}_{1, m}^{R, T_{1}}:=\left[\widetilde{\mathbf{H}}_{1, m}^{B R, 2, T_{1}} \mid \widetilde{\mathbf{H}}_{1, m}^{B R, 3, T_{1}}\right] \in \mathbb{C}^{L N_{R} \times 2 S^{B, T_{1}}}
$$

for RN $m$ in macrocell 1 , and

$$
\widehat{\mathbf{H}}_{2, k}^{U, T_{1}}:=\left[\widetilde{\mathbf{H}}_{2, k}^{B U, 1, T_{1}} \mid \widetilde{\mathbf{H}}_{2, k}^{B U, 3, T_{1}}\right] \in \mathbb{C}^{L N_{U} \times 2 S^{B, T_{1}}}
$$

for UE $k$ in macrocell 2. These matrices are associated with a left nullspace of at least $S^{R}$ and $S^{U}$ dimensions if

$$
L N_{R}-2 S^{B, T_{1}} \geq S^{R}
$$

and

$$
L N_{U}-2 S^{B, T_{1}} \geq S^{U}
$$

respectively. Therefore, to guarantee $S^{R}$ and $S^{U}$ receive dimensions at the RNs and UEs, respectively, $S^{B, T_{1}}$ is derived as

$$
S^{B, T_{1}}=\left\lfloor\min \left(\frac{L N_{R}-S^{R}}{2}, \frac{L N_{U}-S^{U}}{2}\right)\right\rfloor .
$$

The intersecting left nullspace may be found using the SVD on $\widehat{\mathbf{H}}_{n, m}^{R, T_{1}}$ and $\widehat{\mathbf{H}}_{n, k}^{U, T_{1}}$, for RN $m$ and UE $k$ in macrocell $n$, respectively. For example, the SVD of $\widehat{\mathbf{H}}_{n, m}^{R, T_{1}}$ may be written as $\mathbf{U}_{n, m}^{R, T_{1}} \mathbf{S}_{n, m}^{R, T_{1}}\left(\mathbf{V}_{n, m}^{R, T_{1}}\right)^{\mathrm{H}}$, where $\mathbf{U}_{n, m}^{R, T_{1}} \in \mathbb{C}^{L N_{R} \times L N_{R}}$ is the left singular matrix containing, as its columns, the left singular vectors of $\widehat{\mathbf{H}}_{n, m}^{R, T_{1}}$, while $\mathbf{S}_{n, m}^{R, T_{1}} \in \mathbb{R}_{+}^{L N_{R} \times 2 S^{B, T_{1}}}$ is a rectangular diagonal matrix whose diagonal entries are the singular values of $\widehat{\mathbf{H}}_{n, m}^{R, T_{1}}$ placed in descending order, and $\mathbf{V}_{n, m}^{R, T_{1}} \in \mathbb{C}^{2 S^{B, T_{1}} \times 2 S^{B, T_{1}}}$ is the right singular matrix containing, as its columns, the right singular vectors of $\widehat{\mathbf{H}}_{n, m}^{R, T_{1}}$. The intersecting left nullspace may then be obtained as the $\left(L N_{R}-2 S^{B, T_{1}}\right)$ rightmost columns of $\mathbf{U}_{n, m}^{R, T_{1}}$ (corresponding to the zero singular values), and this nullspace is used as the RxBFM, $\mathbf{R}_{n, m}^{R, T_{1}}$, for $\mathrm{RN} m$ in macrocell $n$. A similar procedure is performed for obtaining the RxBFM, $\mathbf{R}_{n, k}^{U, T_{1}}$, for UE $k$ in macrocell $n$ in the first phase, where the $\left(L N_{U}-2 S^{B, T_{1}}\right)$ rightmost columns of the corresponding left singular matrix are selected.

To summarize, the cost of implementing the full-IA protocol in the first transmission phase is the reduction of the number of available spatial transmission streams at each BS from $L N_{B}$ to $S^{B, T_{1}}$. In general, if the values of $S^{R}$ and/or $S^{U}$ are large, the BSs have to substantially reduce the number of transmitted streams in order to accommodate IA. However, it is clear that $S^{B, T_{1}}$ should be higher than 0 to ensure that the
BSs become capable of transmitting. Following this procedure, the $\left(3 S^{B, T_{1}}-S^{R}\right)$ and $\left(3 S^{B, T_{1}}-S^{U}\right)$ total interference signal dimensions received at each $\mathrm{RN}$ and at each UE respectively have each been aligned to $2 S^{B, T_{1}}$ dimensions, leaving $L N_{R}-2 S^{B, T_{1}} \geq S^{R}$ and $L N_{U}-2 S^{B, T_{1}} \geq S^{U}$ receive signal dimensions free from interference at the RNs and UEs, respectively. Thus, IA has been successfully employed for reducing the number of spatial dimensions that the interference signals occupy.

2) Partial-IA Receiver Design: Using this design philosophy, the OCI encountered during the first phase is ignored when designing the RxBFMs. However, because there is no ICI in the first phase since only the BSs are transmitting, there is no need to reduce the number of transmit dimensions at the BSs. Therefore,

$$
S^{B, T_{1}}=L N_{B}
$$

is chosen. Furthermore, the matched filter receiver design is adopted for maximizing the achievable SE [38]. In this case, the SVD is performed on the intra-cell precoded channel matrices, yielding for example

$$
\begin{aligned}
& \widetilde{\mathbf{H}}_{n, m}^{B R, n, T_{1}}=\mathbf{U}_{n, m}^{B R, n, T_{1}} \mathbf{S}_{n, m}^{B R, n, T_{1}}\left(\mathbf{V}_{n, m}^{B R, n, T_{1}}\right)^{\mathrm{H}} \\
& \widetilde{\mathbf{H}}_{n, k}^{B U, n, T_{1}}=\mathbf{U}_{n, k}^{B U, n, T_{1}} \mathbf{S}_{n, k}^{B U, n, T_{1}}\left(\mathbf{V}_{n, k}^{B U, n, T_{1}}\right)^{\mathrm{H}}
\end{aligned}
$$

respectively, and the $S^{R}$ (resp. $S^{U}$ ) leftmost left (thus corresponding to the highest singular values) singular vectors are selected as the RxBFM for the RNs (resp. UEs) in the first phase.

In summary, IA is not required during the first transmission phase of the partial-IA protocol, since the only transmitter within the same cell is the associated BS. Therefore, it is not necessary for the BSs to reduce the number of transmit dimensions available to them for the sake of avoiding interference.

3) Scheduling and Transmitter Design: Having designed the RxBFMs, the effective DL channel matrices can be written as

or

$$
\begin{aligned}
& \overline{\mathbf{H}}_{n, m}^{B R, n, T_{1}}:=\left(\mathbf{R}_{n, m}^{R, T_{1}}\right)^{\mathrm{H}} \widetilde{\mathbf{H}}_{n, m}^{B R, n, T_{1}} \\
& \overline{\mathbf{H}}_{n, k}^{B U, n, T_{1}}:=\left(\mathbf{R}_{n, k}^{U, T_{1}}\right)^{\mathrm{H}} \widetilde{\mathbf{H}}_{n, k}^{B U, n, T_{1}}
\end{aligned}
$$

for RN $m$ and UE $k$ in macrocell $n$, respectively. We term the rows of these matrices as the SMCs of the associated transceivers, since each SMC corresponds to a distinct virtual multiple-input-single-output (MISO) channel between the associated transmitter as well as receiver, and then multiple MISOs can be multiplexed for composing a MIMO channel. A set of SMCs is generated for each of the two transmission phases, and each BS then distributively groups these SMCs according to the semi-orthogonal user selection algorithm ${ }^{3}$, as described in [20], [39], given a semi-orthogonality parameter $\alpha$. For the first transmission phase, up to $\min \left(S^{B, T_{1}}, K L N_{U}+M L N_{R}\right)$ SMCs may be served simultaneously by each BS, while avoiding ICI.

\footnotetext{
${ }^{3}$ This selection method aims for reducing the power loss imposed by the channel inversion operation of the ZFBF matrix [20], [27].
} 
The set of groupings available for BS $n$ is denoted by ${ }^{4} \mathcal{G}^{n}$. The SMCs belonging to group $j$, which are denoted by $\mathcal{E}^{n, j}$, are then the rows of the effective scheduled DL matrix, denoted by $\underline{\mathbf{H}}^{B, n, j, T_{1}}$ for macrocell $n$. In order to avoid ICI between these selected SMCs of group $j$, macrocell $n$ applies the ZFBF matrix $\mathbf{T}^{B, n, j, T_{1}}$, given in (14), as the right channel inverse before using its TP matrix, $\mathbf{A}^{B, n}$, where $\left(\mathbf{W}^{B, n, j, T_{1}}\right)^{\frac{1}{2}}$ is a real-valued diagonal matrix, which normalizes the columns of $\mathbf{T}^{B, n, j, T_{1}}$ for ensuring that the power assigned to each transmission remains unaffected.

$$
\begin{aligned}
\mathbf{T}^{B, n, j, T_{1}} & =\left(\underline{\mathbf{H}}^{B, n, j, T_{1}}\right)^{\mathrm{H}} \\
\times & {\left[\underline{\mathbf{H}}^{B, n, j, T_{1}}\left(\underline{\mathbf{H}}^{B, n, j, T_{1}}\right)^{\mathrm{H}}\right]^{-1}\left(\mathbf{W}^{B, n, j, T_{1}}\right)^{\frac{1}{2}} }
\end{aligned}
$$

The effective end-to-end channel power gains are then given by the squares of the diagonal entries in $\left(\mathbf{W}^{B, n, j, T_{1}}\right)^{\frac{1}{2}}$. For SMC $e_{1}$ in group $j$ of macrocell $n$ corresponding to a direct first phase BS-UE link, the effective channel power gain is denoted by $w_{n, e_{1}}^{B U, n, j, T_{1}}$, while the effective channel power gain of the OCI link, originating from macrocell $n^{\prime}$ serving SMC group $j^{\prime}$ to UE $k$ in macrocell $n$, is obtained from the specific diagonal element of 5

$$
\left|\left(\mathbf{R}_{n, k}^{U, T_{1}}\right)^{\mathrm{H}} \widetilde{\mathbf{H}}_{n, k}^{B U, n^{\prime}, T_{1}} \mathbf{T}^{B, n^{\prime}, j^{\prime}, T_{1}}\right|^{2}
$$

corresponding to SMC $e_{1}$ at UE $k$ of macrocell $n$, and is denoted by $w_{n, e_{1}}^{B U, n^{\prime}, j^{\prime}, T_{1}}$. In the case of the full-IA protocol, all OCI is avoided, thus $w_{n, e_{1}}^{B U, n^{\prime}, j^{\prime}, T_{1}}=0, \forall n^{\prime} \neq n$. The effective channel power gain for the BS-to-RN link, corresponding to SMC-pair ${ }^{6}$ $e$, may be similarly obtained and is denoted by $w_{n, e}^{B R, n, j, T_{1}}$, whereas that of an OCI link is denoted by $w_{n, e}^{B R, n^{\prime}, j^{\prime}, T_{1}}$.

\section{B. Beamforming Design for the Second Phase}

During the second phase, both the BSs and the RNs may transmit. Therefore, in a similar fashion to the first phase, the BS in cell $n$ adopts the precoding matrix $\mathbf{A}^{B, n, T_{2}} \in \mathbb{C}^{L N_{B} \times S^{B, T_{2}}}$, while RN $m$ in cell $n$ adopts the precoding matrix $\mathbf{A}^{R, n, m, T_{2}} \in$ $\mathbb{C}^{L N_{R} \times S^{R}}$, which are again complex-valued matrices having a full column-rank. Additionally, the columns of these TP matrices are normalized. Due to the additional interference imposed by the transmissions of the RNs, it is necessary to reduce the number of transmit dimensions at the BSs even further in order to facilitate IA at the DL receivers. Additionally, note that each TP matrix used at the RNs consist of $S^{R}$ columns, since the information received by each $\mathrm{RN}$ during the first phase must be conveyed to the intended UE. The precoded channel matrices used during the second phase are thus given by (note that the transmitter indices are $n^{\prime}$ and $m^{\prime}$, since these may be inter-cell channel matrices)

$$
\widetilde{\mathbf{H}}_{n, k}^{R U, n^{\prime}, m^{\prime}, T_{2}}:=\mathbf{H}_{n, k}^{R U, n^{\prime}, m^{\prime}} \mathbf{A}^{R, n^{\prime}, m^{\prime}, T_{2}} \in \mathbb{C}^{L N_{U} \times S^{R}}
$$

${ }^{4}$ Note that each group additionally contains the SMCs selected for the second phase, as it will be discussed in Section III.B3.

${ }^{5}$ Note that $|\mathbf{A}|^{2}$ denotes the elementwise squared absolute values of the matrix $\mathbf{A}$ in this work.

${ }^{6}$ Relaying links contain both a SMC for the BS-RN link and a SMC for the RN-UE link and

$$
\widetilde{\mathbf{H}}_{n, k}^{B U, n^{\prime}, T_{2}}:=\mathbf{H}_{n, k}^{B U, n^{\prime}} \mathbf{A}^{B, n^{\prime}, T_{2}} \in \mathbb{C}^{L N_{U} \times S^{B, T_{2}}} .
$$

1) Full-IA Receiver Design: The receiver design used during the second phase depends on whether the BS or a $\mathrm{RN}$ is selected to serve each UE within the same macrocell. Each of the $(1+M)$ possible transmitters may be examined for the sake of finding the most beneficial choice. For example, assuming that BS 1 transmits to UE $k$ during the second phase, the OCI and ICI channel matrices may be concatenated to form (18). However, when assuming for example, that RN 1 of macrocell $n$ transmits to UE $k$, the combined interference matrix is defined by (19).

$$
\begin{aligned}
& \widehat{\mathbf{H}}_{1, k}^{B U, 1, T_{2}}: \\
&= {\left[\widetilde{\mathbf{H}}_{1, k}^{B U, 2, T_{2}}\left|\widetilde{\mathbf{H}}_{1, k}^{B U, 3, T_{2}}\right| \widetilde{\mathbf{H}}_{1, k}^{R U, 1,1, T_{2}}|\ldots| \widetilde{\mathbf{H}}_{1, k}^{R U, 1, M, T_{2}}\right.} \\
&\left|\widetilde{\mathbf{H}}_{1, k}^{R U, 2,1, T_{2}}\right| \ldots\left|\widetilde{\mathbf{H}}_{1, k}^{R U, 2, M, T_{2}}\right| \widetilde{\mathbf{H}}_{1, k}^{R U, 3,1, T_{2}} \mid \ldots \\
&\left.\mid \widetilde{\mathbf{H}}_{1, k}^{R U, 3, M, T_{2}}\right] \\
& \in\left.\mathbb{C}^{L N_{U} \times\left(2 S^{B}, T_{2}\right.}+3 M S^{R}\right) \\
& \widehat{\mathbf{H}}_{n, k}^{R U, n, 1, T_{2}}: \\
&= {\left[\widetilde{\mathbf{H}}_{n, k}^{B U, 1, T_{2}}\left|\widetilde{\mathbf{H}}_{n, k}^{B U, 2, T_{2}}\right| \widetilde{\mathbf{H}}_{n, k}^{B U, 3, T_{2}}\left|\widetilde{\mathbf{H}}_{n, k}^{R U, 1,2, T_{2}}\right| \ldots\right.} \\
& \mid \widetilde{\mathbf{H}}_{n, k}^{R U, 1, M, T_{2}} \\
&\left|\widetilde{\mathbf{H}}_{n, k}^{R U, 2,1, T_{2}}\right| \ldots\left|\widetilde{\mathbf{H}}_{n, k}^{R U, 2, M, T_{2}}\right| \widetilde{\mathbf{H}}_{n, k}^{R U, 3,1, T_{2}} \mid \ldots \\
&\left.\mid \widetilde{\mathbf{H}}_{n, k}^{R U, 3, M, T_{2}}\right] \\
& \in \mathbb{C}^{L N_{U} \times\left[3 S^{B, T_{2}}+(3 M-1) S^{R}\right]}
\end{aligned}
$$

Therefore, in order to guarantee having $S^{U}$ receive dimensions at each UE, we have

$$
S^{B, T_{2}}=\left\lfloor\operatorname { m i n } \left(\frac{L N_{U}-S^{U}-3 M S^{R}}{2},\right.\right.
$$

In both cases described above, the SVD may again be employed for finding the intersecting left nullspace of the precoded interference matrix. The RxBFM, $\mathbf{R}_{n, k}^{U, T_{2}}$, at UE $k$ in macrocell $n$ used during the second phase is then given by the rightmost (thus corresponding to its zero singular values) $L N_{U}-\left(2 S^{B, T_{2}}+3 M S^{R}\right)$ in the left singular matrix of $\widehat{\mathbf{H}}_{1, k}^{B U, 1, T_{2}}$, when the BS is the activated transmitter. By contrast, when assuming that RN 1 is the activated transmitter, the rightmost $\min \left(S^{R}, L N_{U}-\left[3 S^{B, T_{2}}+(3 M-1) S^{R}\right]\right)$ columns in the ordered left singular matrix of $\widehat{\mathbf{H}}_{n, k}^{R U, n, 1, T_{2}}$ specify the RxBFM matrix.

In conclusion, the BSs once again have to reduce the number of spatial transmission streams available to them in order to facilitate IA. In this case, their number is reduced from $L N_{B}$ to $S^{B, T_{2}}$. Additionally, each $\mathrm{RN}$ reduces the number of streams available for them to transmit from $L N_{R}$ to $S_{R}$. On one hand, when the BS is selected as the active transmitter for a particular UE using the full-IA protocol, a 
total of $\left(3 S^{B, T_{2}}+3 M S^{R}-S^{U}\right)$ interference signal dimensions are aligned to $\left(2 S^{B, T_{2}}+3 M S^{R}\right)$ signal dimensions, leaving $L N_{U}-\left(2 S^{B, T_{2}}+3 M S^{R}\right) \geq S^{U}$ signal dimensions free from interference. Thus, IA has been successfully employed. On the other hand, when a RN is selected as the activated transmitter for a particular UE, there is a total of $\left(3 S^{B, T_{2}}+3 M S^{R}-S^{U}\right) \quad$ interference signal dimensions, which are aligned to $\left(3 S^{B, T_{2}}+3 M S^{R}-S^{R}\right)$ signal dimensions. Therefore, IA is only feasible at the UEs if we have $S^{R}>S^{U}$. The constraint given by

$$
L N_{U}-\left(3 S^{B, T_{2}}+3 M S^{R}\right)-S^{R}>S^{R}
$$

is additionally enforced in the full-IA protocol, so that the CCI can still be nulled when $S^{R} \leq S^{U}$ and a $\mathrm{RN}$ is selected as the active transmitter. However, IA is not employed in this case.

2) Partial-IA Receiver Design: Although the effects of OCI are ignored when using this protocol, the ICI must be avoided. Thus, the interference matrix, assuming for example that the BS is the selected transmitter for UE $k$ in macrocell 1 , is then given by

$$
\widehat{\mathbf{H}}_{1, k}^{B U, 1, T_{2}}:=\left[\widetilde{\mathbf{H}}_{1, k}^{R U, 1,1, T_{2}}|\cdots| \widetilde{\mathbf{H}}_{1, k}^{R U, 1, M, T_{2}}\right] \in \mathbb{C}^{L N_{U} \times M S^{R}} .
$$

By contrast, if RN 1 of macrocell $n$ is selected as the transmitter for UE $k$, then the interference matrix is given by

$$
\begin{aligned}
\widehat{\mathbf{H}}_{n, k}^{R U, n, 1, T_{2}} & :=\left[\widetilde{\mathbf{H}}_{n, k}^{B U, 1, T_{2}}\left|\widetilde{\mathbf{H}}_{n, k}^{R U, n, 2, T_{2}}\right| \cdots \mid \widetilde{\mathbf{H}}_{n, k}^{R U, n, M, T_{2}}\right] \\
& \in \mathbb{C}^{L N_{U} \times\left[S^{B, T_{2}}+(M-1) S^{R}\right]},
\end{aligned}
$$

which implies that

$$
S^{B, T_{2}}=L N_{U}-S^{U}-(M-1) S^{R}
$$

is satisfied for ensuring that the UEs are capable of finding approximate RxBFMs, which completely null the ICI.

Thus, UE $k$ may employ the $L N_{U}-M S^{R}$ rightmost left singular columns in $\widehat{\mathbf{H}}_{1, k}^{B U, 1, T_{2}}$ as its RxBFM, when the $\mathrm{BS}$ is the activated transmitter. By contrast, assuming that $\mathrm{RN} 1$ is the activated transmitter, the $\min \left(S^{R}, L N_{U}-\left[S^{B, T_{2}}+(M-1) S^{R}\right]\right)$ rightmost left singular columns in $\widehat{\mathbf{H}}_{n, k}^{R U, n, 1, T_{2}}$ specify the RxBFM.

To summarize, the BSs reduce the spatial streams available to them from $L N_{B}$ to $S^{B, T_{2}}$, while the RNs reduce the number of their spatial streams from $L N_{R}$ to $S^{R}$. On one hand, when the BS is selected as the active transmitter for the partial-IA protocol, a total of $\left(S^{B, T_{2}}+M S^{R}-S^{U}\right)$ interference signal dimensions are aligned to $M S^{R}$ signal dimensions, leaving $L N_{U}-M S^{R} \geq S^{U}$ signal dimensions free from interference. Thus, IA has been successfully employed. On the other hand, when a $\mathrm{RN}$ is selected as the activated transmitter, there are a total of $\left(S^{B, T_{2}}+M S^{R}-S^{U}\right)$ interference signal dimensions, which are aligned to $\left(S^{B, T_{2}}+M S^{R}-S^{R}\right)$ signal dimensions. Therefore, IA is only feasible for $S^{R}>S^{U}$. However, the aforementioned RxBFMs are still capable of nulling the $\mathrm{CCI}$, when a $\mathrm{RN}$ is selected as the active transmitter in the partial-IA protocol and we have $S^{R} \leq S^{U}$. But in this case the constraint given by (21) is not required, since it is already satisfied by (24).

3) Scheduling and Transmitter Design: In a similar fashion to the first phase, the effective DL channel matrices are given by

$$
\overline{\mathbf{H}}_{n, k}^{R U, n, m, T_{2}}:=\left(\mathbf{R}_{n, k}^{U, T_{2}}\right)^{\mathrm{H}} \widetilde{\mathbf{H}}_{n, k}^{R U, n, m, T_{2}}
$$

and

$$
\overline{\mathbf{H}}_{n, k}^{B U, n, T_{2}}:=\left(\mathbf{R}_{n, k}^{U, T_{2}}\right)^{\mathrm{H}} \widetilde{\mathbf{H}}_{n, k}^{B U, n, T_{2}},
$$

when the $\mathrm{BS}$ or $\mathrm{RN} m$ is activated as the transmitter for UE $k$ belonging to macrocell $n$, respectively. The rows of the DL TxBFMs corresponding to each transmitter form the SMCs for that transmitter, and they may be grouped at each BS according to the semi-orthogonal user selection algorithm described above. Furthermore, in the second phase, each BS can select up to $\min \left(S^{B, T_{2}}, K L N_{U}\right)$ SMCs to serve simultaneously while avoiding ICI, whereas each RN may select $\min \left(S^{R}, K L N_{U}\right)$ SMCs. At BS $n$ (or RN $m$ of macrocell $n$ ), the selected SMCs of group $j$ form the rows of its effective scheduled DL matrix, denoted by $\underline{\mathbf{H}}^{B, n, j, T_{2}}$ (or $\underline{\mathbf{H}}^{R, n, m, j, T_{2}}$ ). The ZFBF matrix employed by BS $n$ or by RN $m$ of macrocell $n$ in the second phase is then given by the right inverse (27) or (28), respectively, where the real-valued diagonal matrices of $\left(\mathbf{W}^{B, n, j, T_{2}}\right)^{\frac{1}{2}}$ and $\left(\mathbf{W}^{R, n, m, j, T_{2}}\right)^{\frac{1}{2}}$ are required for normalizing the columns of $\mathbf{T}^{B, n, j, T_{2}}$ and $\mathbf{T}^{R, n, m, j, T_{2}}$, respectively.

$$
\begin{aligned}
& \mathbf{T}^{B, n, j, T_{2}}=\left(\underline{\mathbf{H}}^{B, n, j, T_{2}}\right)^{\mathrm{H}} \\
& \quad \times\left[\underline{\mathbf{H}}^{B, n, j, T_{2}}\left(\underline{\mathbf{H}}^{B, n, j, T_{2}}\right)^{\mathrm{H}}\right]^{-1}\left(\mathbf{W}^{B, n, j, T_{2}}\right)^{\frac{1}{2}} \\
& \mathbf{T}^{R, n, m, j, T_{2}}=\left(\underline{\mathbf{H}}^{R, n, m, j, T_{2}}\right)^{\mathrm{H}} \\
& \quad \times\left[\underline{\mathbf{H}}^{R, n, m, j, T_{2}}\left(\underline{\mathbf{H}}^{R, n, m, j, T_{2}}\right)^{\mathrm{H}}\right]^{-1}\left(\mathbf{W}^{R, n, m, j, T_{2}}\right)^{\frac{1}{2}}
\end{aligned}
$$

The effective channel power gains in the second phase are thus given by the squares of the diagonal entries in $\left(\mathbf{W}^{B, n, j, T_{2}}\right)^{\frac{1}{2}}$ and $\left(\mathbf{W}^{R, n, m, j, T_{2}}\right)^{\frac{1}{2}}$. The effective channel power gain of a BS-UE SMC $e_{2}$ of group $j$ associated with macrocell $n$ and UE $k$ is denoted by $w_{n, e_{2}}^{B U, n, j, T_{2}}$, while the RN-UE effective channel power gain of SMC-pair $e$ associated with RN $m$ of macrocell $n$ and UE $k$ may be denoted by $w_{n, e}^{R U, n, m, j, T_{2}}$. Similar to the first phase, the effective channel power gain of the OCI link originating from the BS of macrocell $n^{\prime}$ serving group $j^{\prime}$ to UE $k$ in macrocell $n$, is obtained from the specific diagonal element of

$$
\left|\left(\mathbf{R}_{n, k}^{U, T_{2}}\right)^{\mathrm{H}} \widetilde{\mathbf{H}}_{n, k}^{B U, n^{\prime}, T_{2}} \mathbf{T}^{B, n^{\prime}, j^{\prime}, T_{2}}\right|^{2}
$$

corresponding to SMC $e_{2}$ at UE $k$ of macrocell $n$, which is denoted by $w_{n, e_{2}}^{B U, n^{\prime}, j^{\prime}, T_{2}}$. On the other hand, the effective channel power gain of the OCI link, originating from $\mathrm{RN} m^{\prime}$ of macrocell $n^{\prime}$ serving group $j^{\prime}$ to UE $k$ of macrocell $n$, is obtained from the specific diagonal element of

$$
\left|\left(\mathbf{R}_{n, k}^{U, T_{2}}\right)^{\mathrm{H}} \widetilde{\mathbf{H}}_{n, k}^{R U, n^{\prime}, m^{\prime}, T_{2}} \mathbf{T}^{R, n^{\prime}, m^{\prime}, j, T_{2}}\right|^{2}
$$


corresponding to SMC $e$ at UE $k$ of macrocell $n$, and is denoted by $w_{n, e}^{R U, n^{\prime}, m^{\prime}, j^{\prime}, T_{2}}$. In the case of the full-IA protocol, all OCI is avoided, thus we have $w_{n, e_{2}}^{B U, n^{\prime}, j^{\prime}, T_{2}}=w_{n, e}^{R U, n^{\prime}, m^{\prime}, j^{\prime}, T_{2}}=$ $0, \forall n^{\prime} \neq n$.

\section{Achievable Spectral Efficiency and Energy Efficiency}

Since we have mathematically decomposed the MIMO channels into effective single-input-single-output (SISO) channels with scalar channel gains given by the diagonal entries of (15), (29), and (30), we may directly employ the Shannon capacity bound for characterizing the achievable ESE performance, rather than relying on bounds derived for MIMO channels [40]. We begin by defining the signal-to-interference-plus-noise ratio (SINR) of the direct link SMCs belonging to group $j$ and intended for UE $k$ of macrocell $n$ during the first and the second phase as

$$
\Gamma_{n, e_{1}}^{B U, n, j, T_{1}}(\mathcal{P}, \mathcal{S})=\frac{w_{n, e_{1}}^{B U, n, j, T_{1}} P_{n, e_{1}}^{B, n, j, T_{1}}}{\Delta \gamma\left(N_{0} L W+I_{n, e_{1}}^{U, T_{1}}\right)}
$$

and

$$
\Gamma_{n, e_{2}}^{B U, n, j, T_{2}}(\mathcal{P}, \mathcal{S})=\frac{w_{n, e_{2}}^{B U, n, j, T_{2}} P_{n, e_{2}}^{B, n, j, T_{2}}}{\Delta \gamma\left(N_{0} L W+I_{n, e_{2}}^{U, T_{2}}\right)},
$$

respectively, where the total received $\mathrm{OCI}$ in the first and second phase has been denoted by (33) and (34), respectively, where $\mathcal{M}(e)$ is a function of $e$, representing the $\mathrm{RN}$ index (similar to $m$ used before) associated with the SMC-pair $e$. For simplicity ${ }^{7}$, the interference that was not avoided using IA is treated as noise.

$$
\begin{aligned}
& I_{n, e_{1}}^{U, T_{1}}(\mathcal{P}, \mathcal{S})=\sum_{\substack{n^{\prime}=1 \\
n^{\prime} \neq n}} \sum_{j^{\prime} \in \mathcal{G}^{n^{\prime}}} s^{n^{\prime}, j^{\prime}} w_{n, e_{1}}^{B U, n^{\prime}, j^{\prime}, T_{1}} \\
& \quad \times\left[\sum_{e_{1}^{\prime} \in \mathcal{E}^{n^{\prime}, j^{\prime}}} P_{n^{\prime}, e_{1}^{\prime}}^{B, n^{\prime}, j^{\prime}, T_{1}}+\sum_{e^{\prime} \in \mathcal{E}^{n^{\prime}, j^{\prime}}} P_{n^{\prime}, e^{\prime}, j^{\prime}, T_{1}}^{B, n^{\prime}}\right] \\
& I_{n, e_{2}}^{U, T_{2}}(\mathcal{P}, \mathcal{S})=\sum_{\substack{n^{\prime}=1 \\
n^{\prime} \neq n}} \sum_{j^{\prime} \in \mathcal{G}^{n^{\prime}}} s^{n^{\prime}, j^{\prime}} \\
& \quad \times\left[w_{n, e_{2}}^{B U, n^{\prime}, j^{\prime}, T_{2}} \sum_{e_{2}^{\prime} \in \mathcal{E}^{n^{\prime}, j^{\prime}}} P_{n^{\prime}, e_{2}^{\prime}, j^{\prime}, T_{2}}^{B, n^{\prime}}\right] \\
& \left.\quad+\sum_{e^{\prime} \in \mathcal{E}^{n^{\prime}, j^{\prime}}} w_{n, e_{2}}^{R U, n^{\prime}, \mathcal{M}\left(e^{\prime}\right), j^{\prime}, T_{2}} P_{n, e^{\prime}}^{R, n^{\prime}, \mathcal{M}\left(e^{\prime}\right), j^{\prime}, T_{2}}\right]
\end{aligned}
$$

The set $\mathcal{P}$ contains the power control variables denoted by $P_{n, e_{1}}^{B, n, j, T_{1}}, P_{n, e}^{B, n, j, T_{1}}, P_{n, e_{2}}^{B, n, j, T_{2}}$, and $P_{n, e}^{R, n, m, j, T_{2}}, \forall n, e_{1}, e_{2}, e$. On the other hand, the set $\mathcal{S}$ contains the group selection indicator variables, $s^{n, j}, \forall n, j$, where $s^{n, j}=1$, when the SMC group $j$ has been selected for macrocell $n$, and $s^{n, j}=0$ otherwise. The total noise power across all subcarrier blocks is given by $N_{0} L W$, while $\Delta \gamma$ is the signal-to-noise ratio (SNR) difference between the SNR at the discrete-input-continuous-output memoryless channel (DCMC) capacity and the actual SNR required by the specific modulation and coding schemes of the practical physical layer transceivers employed [41].

${ }^{7}$ If the level of interference is strong enough, then more sophisticated methods, such as multiuser detection, may be employed.
The SINR of the BS-RN SMC $e$ belonging to group $j$ of macrocell $n$ and intended for RN $m$ may be expressed as

$$
\Gamma_{n, e}^{B R, n, j, T_{1}}(\mathcal{P}, \mathcal{S})=\frac{w_{n, e}^{B R, n, j, T_{1}} P_{n, e}^{B, n, j, T_{1}}}{\Delta \gamma\left(N_{0} L W+I_{n, e}^{R, T_{1}}\right)},
$$

while the SINR of the corresponding RN-UE link may be formulated as

$$
\Gamma_{n, e}^{R U, n, m, j, T_{2}}(\mathcal{P}, \mathcal{S})=\frac{w_{n, e}^{R U, n, m, j, T_{2}} P_{n, e}^{R, n, m, j, T_{2}}}{\Delta \gamma\left(N_{0} L W+I_{n, e}^{U, T_{2}}\right)},
$$

where the total received OCI of the BS-RN and RN-UE links is given by (37) and (38), respectively.

$$
\begin{aligned}
& I_{n, e}^{R, T_{1}}(\mathcal{P}, \mathcal{S})=\sum_{\substack{n^{\prime}=1 \\
n^{\prime} \neq n}} \sum_{j^{\prime} \in \mathcal{G}^{n^{\prime}}} s^{n^{\prime}, j^{\prime}} w_{n, e}^{B R, n^{\prime}, j^{\prime}, T_{1}} \\
& \quad \times\left[\sum_{e_{1}^{\prime} \in \mathcal{E}^{n^{\prime}, j^{\prime}}} P_{n^{\prime}, e_{1}^{\prime}}^{B, n^{\prime}, j^{\prime}, T_{1}}+\sum_{e^{\prime} \in \mathcal{E}^{n^{\prime}, j^{\prime}}} P_{n^{\prime}, e^{\prime}}^{B, n^{\prime}, j^{\prime}, T_{1}}\right] \\
& I_{n, e}^{U, T_{2}}(\mathcal{P}, \mathcal{S})=\sum_{\substack{n^{\prime}=1 \\
n^{\prime} \neq n}} \sum_{j^{\prime} \in \mathcal{G}^{n^{\prime}}} s^{n^{\prime}, j^{\prime}} \\
& \quad \times\left[w_{n, e}^{B U, n^{\prime}, j^{\prime}, T_{2}} \sum_{e_{2}^{\prime} \in \mathcal{E}^{n^{\prime}, j^{\prime}}} P_{n^{\prime}, e_{2}^{\prime}}^{B, n^{\prime}, j^{\prime}, T_{2}}\right] \\
& \left.+\sum_{e^{\prime} \in \mathcal{E}^{n^{\prime}, j^{\prime}}} w_{n, e}^{R U, n^{\prime}, \mathcal{M}\left(e^{\prime}\right), j^{\prime}, T_{2}} P_{n^{\prime}, e^{\prime}}^{R, n^{\prime}, \mathcal{M}\left(e^{\prime}\right), j^{\prime}, T_{2}}\right]
\end{aligned}
$$

The achievable SE of the direct first and second phase transmissions can be respectively written as

$$
C_{n, e_{1}}^{B U, n, j, T_{1}}(\mathcal{P}, \mathcal{S})=\frac{1}{2} \log _{2}\left(1+\Gamma_{n, e_{1}}^{B U, n, j, T_{1}}\right)
$$

and

$$
C_{n, e_{2}}^{B U, n, j, T_{2}}(\mathcal{P}, \mathcal{S})=\frac{1}{2} \log _{2}\left(1+\Gamma_{n, e_{2}}^{B U, n, j, T_{2}}\right)
$$

where the pre-log factor of $\frac{1}{2}$ accounts for the fact that the transmission period has been split into two phases. When using the DF protocol, the achievable SE of the relaying link is limited by the weaker of the BS-RN and RN-UE links [5], which is given by

$$
\begin{aligned}
C_{n, e}^{B R U, n, m, j}(\mathcal{P}, \mathcal{S})=\min & {\left[\frac{1}{2} \log _{2}\left(1+\Gamma_{n, e}^{B R, n, j, T_{1}}\right),\right.} \\
& \left.\frac{1}{2} \log _{2}\left(1+\Gamma_{n, e}^{R U, n, m, j, T_{2}}\right)\right] .
\end{aligned}
$$

Thus the total achievable SE of macrocell $n$ is given by

$$
\begin{aligned}
& C_{T}^{n}(\mathcal{P}, \mathcal{S})=\sum_{j \in \mathcal{G}^{n}} s^{n, j}\left[\sum_{e_{1} \in \mathcal{E}^{n, j}} C_{n, e_{1}}^{B U, n, j, T_{1}}\right. \\
& \left.\quad+\sum_{e_{2} \in \mathcal{E}^{n, j}} C_{n, e_{2}}^{B U, n, j, T_{2}}+\sum_{e \in \mathcal{E}^{n, j}} C_{n, e}^{B R U, n, \mathcal{M}(e), j}\right]
\end{aligned}
$$


Furthermore, we simplified the energy dissipation model of [42] in order to formulate the total energy dissipation in macrocell $n$ as

$$
\begin{aligned}
& P_{T}^{n}(\mathcal{P}, \mathcal{S})=\left(P_{C}^{B}+M P_{C}^{R}\right) \\
& \quad+\frac{1}{2} \sum_{j \in \mathcal{G}^{n}} s^{n, j}\left[\xi^{B}\left(\sum_{e_{1} \in \mathcal{E}^{n, j}} P_{n, e_{1}}^{B, n, j, T_{1}}+\sum_{e_{2} \in \mathcal{E}^{n, j}} P_{n, e_{2}}^{B, n, j, T_{2}}\right)\right. \\
& \left.\quad+\sum_{e \in \mathcal{E}^{n, j}}\left(\xi^{B} P_{n, e}^{B, n, j, T_{1}}+\xi^{R} P_{n, e}^{R, n, \mathcal{M}(e), j, T_{2}}\right)\right] .
\end{aligned}
$$

The effect of the number of TAs, of the energy dissipation of the RF as well as of the baseband circuits, and the efficiencies of the power amplifier, feeder cables, cooling system, mains power supply, and converters has been accounted for in the fixed energy dissipation terms of $P_{C}^{B}$ and $P_{C}^{R}$, while the transmit power dependent terms $\xi^{B}$ and $\xi^{R}$ are associated with the BS $n$ and its RNs, respectively.

Thus, the ESE of macrocell $n$ is given by

$$
\eta_{E}^{n}(\mathcal{P}, \mathcal{S})=\frac{C_{T}^{n}(\mathcal{P}, \mathcal{S})}{P_{T}^{n}(\mathcal{P}, \mathcal{S})}
$$

In the sequel, our aim is to maximize (44) for each macrocell $n$ by the careful optimization of the variables contained within $\mathcal{P}$ and $\mathcal{S}$. We define the average ESE of the multicell system as

$$
\eta_{E}(\mathcal{P}, \mathcal{S})=\frac{1}{3} \sum_{n=1}^{3} \eta_{E}^{n}(\mathcal{P}, \mathcal{S}),
$$

so that the average ESE of the system can be optimized by individually maximizing each macrocell's ESE, as it will be discussed in the following.

\section{Optimization Problem Formulation AND SOLUTION ALGORITHM}

In this section, our aim is to optimize the OF (45). We formally describe the optimization problem as (46)-(52). To elaborate, (45) is maximized by appropriately optimizing the decision variables contained within the sets $\mathcal{P}$ and $\mathcal{S}$. The constraint (47) ensures that each macrocell only serves a single SMC group, thus the ICI is completely avoided. The constraints (48)-(50) require that none of the transmitters exceeds its maximum transmission power constraint. Observe that two constraints are needed for each BS, since each BS transmits in both phases, whereas the RNs only transmit during the second phase. Furthermore, the constraint (51) reflects the binary constraint imposed on the $s^{n, j}$ variables, while the constraints (52) ensures that the power control variables are non-negative.

$$
\underset{\mathcal{P}, \mathcal{S}}{\operatorname{maximize}}(45)
$$

$$
\begin{aligned}
& \text { subject to } \sum_{j \in \mathcal{G}^{n}} s^{n, j} \leq 1, \forall n, \\
& \sum_{j \in \mathcal{G}^{n}} s^{n, j}\left[\sum_{e_{1} \in \mathcal{E}^{n, j}} P_{n, e_{1}}^{B, n, j, T_{1}}+\sum_{e \in \mathcal{E}^{n, j}} P_{n, e}^{B, n, j, T_{1}}\right] \\
& \leq P_{\max }^{B}, \forall n, \\
& \sum_{j \in \mathcal{G}^{n}} s^{n, j} \sum_{e_{2} \in \mathcal{E}^{n, j}} P_{n, e_{2}}^{B, n, j, T_{2}} \\
& \leq P_{\max }^{B}, \forall n, \\
& \sum_{j \in \mathcal{G}^{n}} s^{n, j} \sum_{\substack{e \in \mathcal{E}^{n, j} \\
\mathcal{M}^{n}(e)=m}} P_{n, e}^{R, n, m, j, T_{2}} \\
& \leq P_{\max }^{R}, \forall n, m, \\
& s^{n, j} \in\{0,1\}, \forall n, j, \\
& P_{n, e_{1}}^{B, n, j, T_{1}}, P_{n, e}^{B, n, j, T_{1}}, P_{n, e_{2}}^{B, n, j, T_{2}}, P_{n, e}^{R, n, m, j, T_{2}} \\
& \geq 0, \forall n, j, e_{1}, e_{2}, e
\end{aligned}
$$

\section{A. Concave Problem Formulation}

Observe that in the full-IA protocol, the OCI terms are negligible or zero, if perfect CSI is available, while in the partial-IA protocol, the OCI terms are intentionally ignored. Therefore, each macrocell's ESE is independent of the decision variables associated with other macrocells, and the optimization problem can be decomposed and solved distributively, where each macrocell optimizes its own ESE. It can be readily proven that the $\mathrm{OF}$ is nonlinear and involves binary variables. Thus, the optimization problem of (46)-(52) is a mixed integer nonlinear programming (MINLP) problem, which are typically solved using high-complexity branch-and-bound methods [43]. In order to mitigate the computational burden of finding a solution to (46)-(52), we relax ${ }^{8}$ the binary constraint imposed on the variables $s^{n, j}$ by replacing the constraint (51) with

$$
0 \leq s^{n, j} \leq 1, \quad \forall n, j .
$$

${ }^{8}$ In [44], such a relaxation results in a time-sharing solution regarding each subcarrier block. In this work, this relaxation may be viewed as time-sharing of each subcarrier block, as multiple SMC groups can then occupy a fraction of each subcarrier block in time. Naturally, the relaxation means that we do not accurately solve the original problem of (46)-(52). In fact, since we have expanded the space of feasible solutions, solving the relaxed problem results in an upper bound of the optimal objective value of the original problem. However, the algorithm devised in this paper for obtaining the optimal solution to the relaxed problem will only retain integer values of the relaxed variables. Therefore, the algorithm essentially maximizes a lower bound of the relaxed problem. Having said that, as shown in [25], [45], [46], the optimal solution to the original problem is still obtained with high probability when using the dual decomposition method on the relaxed problem (as in this work) as the number of subcarriers tends to infinity. It was shown that 8 subcarriers is sufficient for this to be true in the context of [47], while we have shown that 2 subcarriers is sufficient in the context of [25].

$$
t^{n}=\frac{1}{\left(P_{C}^{B}+M P_{C}^{R}\right)+\frac{1}{2} \sum_{j \in \mathcal{G}^{n}}\left[\xi^{B} \sum_{e_{1} \in \mathcal{E}^{n, j}} \widetilde{P}_{n, e_{1}}^{B, n, j, T_{1}}+\sum_{e_{2} \in \mathcal{E}^{n, j}} \widetilde{P}_{n, e_{2}}^{B, n, j, T_{2}}+\sum_{e \in \mathcal{E}^{n, j}} \xi^{B} \widetilde{P}_{n, e}^{B, n, j, T_{1}}+\xi^{R} \widetilde{P}_{n, e}^{R, n, m, j, T_{2}}\right]}
$$


Additionally, we introduce the auxiliary variables

$$
\begin{aligned}
\widetilde{P}_{n, e_{1}}^{B, n, j, T_{1}} & =t^{n} s^{n, j} P_{n, e_{1}}^{B, j, T_{1}}, \\
\widetilde{P}_{n, e}^{B, n, j, T_{1}} & =t^{n} s^{n, j} P_{n, n}^{B, j, T_{1}}, \\
\widetilde{P}_{n, e_{2}}^{B, n, j, T_{2}} & =t^{n} s^{n, j} P_{n, e_{2}}^{B, n, T_{2}}, \\
\widetilde{P}_{n, e}^{R, n, m, j, T_{2}} & =t^{n} s^{n, j} P_{n, e}^{R, n, m, j, T_{2}}, \\
\widetilde{s}^{n, j} & =t^{n} s^{n, j}, \forall n, j, e_{1}, e_{2}, e,
\end{aligned}
$$

where $t^{n}$ is given by (54), shown at the bottom of the previous page. Note that we have applied the Charnes-Cooper variable transformation [30] using $t^{n}$. Furthermore, the auxiliary SE variables $\widetilde{C}_{n, e_{1}}^{B U, n, j, T_{1}}, \widetilde{C}_{n, e_{2}}^{B U, n, j, T_{2}}$ and $\widetilde{C}_{n, e}^{B R U, n, m, j}$ are introduced, so that we may rewrite the optimization problem of (46)-(52) in the hypograph form [31] given by (60)-(71), $\forall n$, where $\widetilde{\mathcal{P}}^{n}, \widetilde{\mathcal{S}}^{n}$ and $\widetilde{\mathcal{C}}^{n}$ denote the variable sets containing the auxiliary variables that are associated with macrocell $n$.

$$
\begin{aligned}
& \underset{\widetilde{\mathcal{P}}^{n}, \widetilde{\mathcal{S}}^{n}, \widetilde{\mathcal{C}^{n}}}{\operatorname{maximize}} \sum_{j \in \mathcal{G}^{n}}\left[\sum_{e_{1} \in \mathcal{E}^{n, j}} \widetilde{C}_{n, k, e_{1}}^{B U, n, j, T_{1}}+\sum_{e_{2} \in \mathcal{E}^{n, j}} \widetilde{C}_{n, k, e_{2}}^{B U, n, j, T_{2}}\right. \\
& \left.+\sum_{e \in \mathcal{E}^{n, j}} \widetilde{C}_{n, k, e}^{B R U, n, \mathcal{M}(e), j}\right] \\
& \frac{\widetilde{s}^{n, j}}{2} \log _{2}\left(1+\frac{w_{n, e_{1}}^{B U, n, T_{1}} \widetilde{P}_{n, e_{1}}^{B, n, j, T_{1}}}{\widetilde{s}^{n, j} \Delta \gamma N_{0} L W}\right) \\
& \geq \widetilde{C}_{n, e_{1}}^{B U, n, j, T_{1}}, \forall j, e_{1}, \\
& \frac{\widetilde{s}^{n, j}}{2} \log _{2}\left(1+\frac{w_{n, e_{2}}^{B U, n, j, T_{2}} \widetilde{P}_{n, e_{2}}^{B, n, j, T_{2}}}{\widetilde{s}^{n, j} \Delta \gamma N_{0} L W}\right) \\
& \geq \widetilde{C}_{n, e_{2}}^{B U, n, T_{2}}, \forall j, e_{2}, \\
& \frac{\widetilde{s}^{n, j}}{2} \log _{2}\left(1+\frac{w_{n, e}^{B R, n, j, T_{1}} \widetilde{P}_{n, e}^{B, n, j, T_{1}}}{\widetilde{s}^{n, j} \Delta \gamma N_{0} L W}\right) \\
& \geq \widetilde{C}_{n, e}^{B R U, n, \mathcal{M}(e), j}, \forall j, e, \\
& \frac{\widetilde{s}^{n, j}}{2} \log _{2}\left(1+\frac{w_{n, e}^{R U, n, \mathcal{M}(e), j, T_{2}} \widetilde{P}_{n, e}^{R, n, \mathcal{M}(e), j, T_{2}}}{\widetilde{s}^{n, j} \Delta \gamma N_{0} L W}\right) \\
& \geq \widetilde{C}_{n, e}^{B R U, n, \mathcal{M}(e), j}, \forall j, e, \\
& \sum_{j \in \mathcal{G}^{n}} \widetilde{s}^{n, j} \leq t^{n} \\
& \sum_{j \in \mathcal{G}^{n}}\left[\sum_{e_{1} \in \mathcal{E}^{n, j}} \widetilde{P}_{n, e_{1}}^{B, n, j, T_{1}}+\sum_{e \in \mathcal{E}^{n, j}} \widetilde{P}_{n, e}^{B, n, j, T_{1}}\right] \\
& \leq t^{n} \cdot P_{\max }^{B}, \\
& \sum_{j \in \mathcal{G}^{n}} \sum_{e_{2} \in \mathcal{E}^{n, j}} \widetilde{P}_{n, e_{2}}^{B, n, j, T_{2}} \leq t^{n} \cdot P_{\max }^{B}, \\
& \sum_{j \in \mathcal{G}^{n}} \sum_{\substack{e \in \mathcal{E}^{n, j} \\
\mathcal{M}(e)=m}} \widetilde{P}_{n, e}^{R, n, m, j, T_{2}} \leq t^{n} \cdot P_{\max }^{R}, \forall m, \\
& 0 \leq \widetilde{s}^{n, j} \leq t, \quad \forall j, \\
& \widetilde{P}_{n, e_{1}}^{B, n, j, T_{1}}, \widetilde{P}_{n, e_{2}}^{B, n, j, T_{2}}, \widetilde{P}_{n, e}^{B, n, j, T_{1}} \widetilde{P}_{n, e}^{R, n, m, j, T_{2}} \\
& \geq 0, \forall j, e_{1}, e_{2}, e, \\
& t^{n} \cdot\left(P_{C}^{B}+M \cdot P_{C}^{R}\right) \\
& +\frac{1}{2} \sum_{j \in \mathcal{G}^{n}}\left[\xi^{B} \sum_{e_{1} \in \mathcal{E}^{n, j}} \widetilde{P}_{n, e_{1}}^{B, n, j, T_{1}}+\sum_{e_{2} \in \mathcal{E}^{n, j}} \widetilde{P}_{n, e_{2}}^{B, n, j, T_{2}}\right.
\end{aligned}
$$

To elaborate further, the constraints (61) and (62) ensure that the auxiliary SE variables given by $\widetilde{C}_{n, e_{1}}^{B U, n, j, T_{1}}$ and $\widetilde{C}_{n, e_{2}}^{B U, n, j, T_{2}}$ do not exceed the direct link SEs obtained from (39) and (40), respectively, while the constraints (63) and (64) have to be combined to guarantee that (41) is adhered to. The constraints (65)-(70) are simply the equivalents of the constraints (47)-(52), when employing the auxiliary variables, while the constraint (71) is the result of the Charnes-Cooper variable transformation [30]. Finally, the OF (60) defines the ESE of macrocell $n$.

Let us now aim for proving that (60)-(71) is a concave maximization problem. It can be readily shown that the OF (60) is linear, hence concave. Similarly, the constraints (65)-(71) are all linear. Therefore, what remains for us to prove is that the constraints (61)-(64) are all convex. Observe that the constraints (61)-(64) are all of the form $\frac{s}{2} \log _{2}\left(1+\frac{a P}{s}\right) \geq C$, where the decision variables are $s, P$ and $C$, while $a$ is some constant. It is plausible that $(1+a P)$ is linear. The function composition of $\frac{1}{2} \log _{2}(1+a P)$ is concave [31] and the perspective transformation [31], giving $\frac{s}{2} \log _{2}\left(1+\frac{a P}{s}\right)$, preserves concavity. Finally, rewriting the previous inequality as $C-\frac{s}{2} \log _{2}\left(1+\frac{a P}{s}\right) \leq 0$ clearly shows that it is indeed a convex constraint. Thus, we have proven that $(60)-(71)$ is a concave programming problem, which may be solved using efficient algorithms. Let us now proceed with the portrayal of the algorithm employed in this work for solving the above problem.

\section{B. Solution Algorithm}

Observe that the optimization problem of (60)-(71) is akin to a sum-rate maximization problem, which is optimally solved using the well-known water-filling method [21]. From our previous work [25]-[27] using dual decomposition [37], we may deduce that the optimal (denoted by a superscript asterisk) values for $\widetilde{P}_{n, e_{1}}^{B, n, j, T_{1}}$ and $\widetilde{P}_{n, e_{2}}^{B, n, j, T_{2}}$ are respectively given by

$\widetilde{P}_{n, e_{1}}^{B, n, j, T_{1} *}=\widetilde{s}^{n, j}\left[\frac{1}{\left(\xi^{B} \mu^{*}+2 \lambda^{n, T_{1} *}\right) \ln 2}-\frac{\Delta \gamma N_{0} L W}{w_{n, e_{1}}^{B U, n, j, T_{1}}}\right]$

and

$\widetilde{P}_{n, e_{2}}^{B, n, j, T_{2} *}=\widetilde{s}^{n, j}\left[\frac{1}{\left(\xi^{B} \mu^{*}+2 \lambda^{n, T_{2} *}\right) \ln 2}-\frac{\Delta \gamma N_{0} L W}{w_{n, e_{2}}^{B U, n, j, T_{2}}}\right]^{+}$,

where $\widetilde{s}^{n, j}$ is yet to be determined, while $[\cdot]^{+}$is equivalent to $\max (0, \cdot)$. Furthermore, $\mu^{*}$ is the optimal Lagrangian dual variable [31] associated with the constraint (71), while $\lambda^{n, T_{1} *}$ and $\lambda^{n, T_{2} *}$ are respectively the optimal Lagrangian dual variables associated with the constraints (66) and (67) for macrocell $n$. The optimal Lagrangian dual variables are chosen to satisfy the constraints (66) - (68) with equality, and are found using the subgradient algorithm [37].

It may be shown that the power control variables of the relaying links may be formulated as

$$
\widetilde{P}_{n, e}^{B, n, j, T_{1}}=\widetilde{s}^{n, j}\left[\frac{1}{\left(\xi^{B} \mu^{*}+2 \lambda^{n, T_{1} *}\right) \ln 2}-\frac{\Delta \gamma N_{0} L W}{w_{n, e}^{B R, n, j, T_{1}}}\right]^{+}
$$


and

$$
\begin{aligned}
\widetilde{P}_{n, e}^{R, n, \mathcal{M}(e), j, T_{2}}= & \widetilde{s}^{n, j}\left[\frac{1}{\left(\xi^{R} \mu^{*}+2 \nu^{n, \mathcal{M}(e), T_{2} *}\right) \ln 2}\right. \\
& \left.-\frac{\Delta \gamma N_{0} L W}{w_{n, e}^{R U, n, \mathcal{M}(e), j, T_{2}}}\right]^{+},
\end{aligned}
$$

where $\nu^{n, \mathcal{M}(e), T_{2} *}$ is the optimal Lagrangian dual variable associated with the constraint (68) for $\mathrm{RN} \mathcal{M}(e)$ belonging to macrocell $n$. Since the attainable SE of a relaying link is limited by the weaker of the BS-RN and RN-UE links, there is no need to transmit at a higher power than necessary, if the other link is unable to support the higher SE. Thus, the optimal power control variables for the relaying link are given by

$$
\begin{aligned}
\widetilde{P}_{n, e}^{B, n, j, T_{1} *}= & \min \left(\frac{w_{n, e}^{R U, n, \mathcal{M}(e), j, T_{2}}}{w_{n, e}^{B R, n, j, T_{1}}}\right. \\
& \left.\times \widetilde{P}_{n, e}^{R, n, \mathcal{M}(e), j, T_{2}}, \widetilde{P}_{n, e}^{B, n, j, T_{1}}\right)
\end{aligned}
$$

and

$$
\begin{aligned}
\widetilde{P}_{n, e}^{R, n, \mathcal{M}(e), j, T_{2} *}= & \min \left(\frac{w_{n, e}^{B R, n, j, T_{1}}}{w_{n, e}^{R U, n, \mathcal{M}(e), j, T_{2}}}\right. \\
& \left.\times \widetilde{P}_{n, e}^{B, n, j, T_{1}}, \widetilde{P}_{n, e}^{R, n, \mathcal{M}(e), j, T_{2}}\right) .
\end{aligned}
$$

Thus, the maximum values of $\widetilde{C}_{n, e_{1}}^{B U, n, j, T_{1}}, \widetilde{C}_{n, e_{2}}^{B U, n, j, T_{2}}$ and $\widetilde{C}_{n, e}^{B R U, n, \mathcal{M}(e), j}$ are given by

$$
\begin{aligned}
\widetilde{C}_{n, e}^{B U, n, j, T_{1}} & =\frac{\widetilde{s}^{n, j}}{2} \log _{2}\left(1+\frac{w_{n, e}^{B U, n, j, T_{1}} \widetilde{P}_{n, e}^{B, n, j, T_{1}}}{\Delta \gamma N_{0} L W}\right), \\
\widetilde{C}_{n, e}^{B U, n, j, T_{2}} & =\frac{\widetilde{s}^{n, j}}{2} \log _{2}\left(1+\frac{w_{n, e}^{B U, n, j, T_{2}} \widetilde{P}_{n, e}^{B, n, j, T_{2}}}{\Delta \gamma N_{0} L W}\right)
\end{aligned}
$$

and (80), where the value of $\widetilde{s}^{n, j}$ is not yet known

$$
\begin{aligned}
\widetilde{C}_{n, e}^{B R U, n, \mathcal{M}(e), j} & \\
= & \frac{\widetilde{s}^{n, j}}{2} \log _{2}\left(1+\frac{w_{n, e}^{B R, n, j, T_{1}} \widetilde{P}_{n, e}^{B, n, j, T_{1}}}{\Delta \gamma N_{0} L W}\right) \\
& =\frac{\widetilde{s}^{n, j}}{2} \log _{2}\left(1+\frac{w_{n, e}^{R U, n, \mathcal{M}(e), j, T_{2}} \widetilde{P}_{n, e}^{R, n, \mathcal{M}(e), j, T_{2}}}{\Delta \gamma N_{0} L W}\right) .
\end{aligned}
$$

However, regardless of the exact value of $\widetilde{s}^{n, j}$, macrocell $n$ may choose the specific SMC group $j$ that obtains the highest value of

$$
\begin{aligned}
\sum_{e_{1} \in \mathcal{E}^{n, j}} \widetilde{C}_{n, e_{1}}^{B U, n, j, T_{1}} & \\
& +\sum_{e_{2} \in \mathcal{E}^{n, j}} \widetilde{C}_{n, e_{2}}^{B U, n, j, T_{2}}+\sum_{e \in \mathcal{E}^{n, j}} \widetilde{C}_{n, e}^{B R U, n, \mathcal{M}(e), j}
\end{aligned}
$$

in order to maximize the OF (60) by setting $\widetilde{s}^{n, j}=t^{n}$, where the value of $t^{n}$ is not yet known. As a result, the SMC groups $j^{\prime} \neq j$ are not chosen and we may set $\widetilde{s}^{n, j^{\prime}}=\widetilde{P}_{n, e_{1}}^{B, n, j^{\prime}, T_{1}}=$ $\widetilde{P}_{n, e_{2}}^{B, n, j^{\prime}, T_{2}}=\widetilde{P}_{n, e}^{B, n, j^{\prime}, T_{1}}=\widetilde{P}_{n, e}^{R, n, \mathcal{M}(e), j^{\prime}, T_{2}}=\widetilde{C}_{n, e_{1}}^{B U, n, j^{\prime}, T_{1}}=$ $\widetilde{C}_{n, e_{2}}^{B U, n, j^{\prime}, T_{2}}=\widetilde{C}_{n, e}^{B R U, n, \mathcal{M}(e), j^{\prime}}=0, \forall e_{1}, e_{2}, e, j^{\prime} \neq j$.
TABLE I

The ESEM ALgorithm BASED ON DUAL DeComposition AND the SUBGRADIENT METHOD

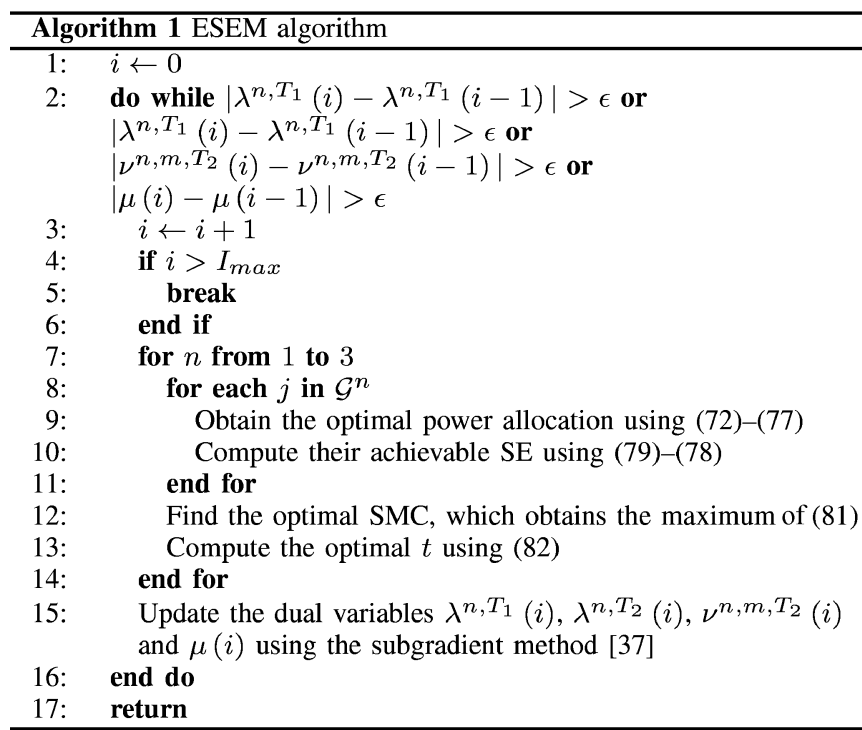

The optimal value of $t^{n}$ is then given by

$$
\begin{aligned}
t^{n}= & \left(P_{C}^{B}+M \cdot P_{C}^{R}+\sum_{j \in \mathcal{G}^{n}} \frac{1}{2 \widetilde{s}^{n, j}}\right. \\
& \times\left[\xi^{B} \sum_{e_{1} \in \mathcal{E}^{n, j}} \widetilde{P}_{n, e_{1}}^{B, n, j, T_{1}}+\sum_{e_{2} \in \mathcal{E}^{n, j}} \widetilde{P}_{n, e_{2}}^{B, n, j, T_{2}}\right. \\
& \left.\left.+\sum_{e \in \mathcal{E}^{n, j}} \xi^{B} \widetilde{P}_{n, e}^{B, n, j, T_{1}}+\xi^{R} \widetilde{P}_{n, e}^{R, n, m, j, T_{2}}\right]\right)^{-1} .
\end{aligned}
$$

Observe that this is possible, since (82) is only dependent on the dual variables. Furthermore, determining the value of $t^{n}$ gives the values of $\widetilde{s}^{n, j}, \widetilde{P}_{n, e_{1}}^{B, n, j, T_{1}}, \widetilde{P}_{n, e_{2}}^{B, n, j, T_{2}}, \widetilde{P}_{n, e}^{B, n, j, T_{1}}$ and $\widetilde{P}_{n, e}^{R, n, m, j, T_{2}}$ for the selected SMC group.

By following the above derivations, the constraints (61)-(65) and (69)-(71) are implicitly satisfied and there is no need to introduce dual variables for them. This ESEM solution algorithm may be implemented distributively, and iterates between obtaining the optimal primal variables and applying the subgradient method [37] for updating the dual variables, until the change in the dual variable values becomes less than $\epsilon$ or the maximum number of iterations, $I_{\max }$, has been reached. The ESEM algorithm is summarized in Table I, where $\lambda^{n, T_{1}}(i), \lambda^{n, T_{2}}(i), \nu^{n, m, T_{2}}(i)$ and $\mu(i)$ indicate the value of their respective dual variables at the $i$ th iteration.

\section{NUMERICAL RESUlts AND DisCUSSIONS}

This section presents the numerical results 9 obtained, when the solution algorithm presented in Section IV.B is employed for the ESEM problem of (60)-(71), where the simulation parameters are given in Table II. Furthermore, we employed the path-

${ }^{9}$ In all cases, the step sizes and the initial values of the dual variables described in Section IV.B are empirically optimized so that the algorithm converges in as few iterations as possible, although the exact analytical method for achieving this still remains an open issue. In our experience, the algorithm converges within just 10 iterations when carefully chosen step sizes are employed, regardless of the size of the problem. 
TABLE II

Simulation Parameters Used to Obtain All Results in This Section UNLESS OTHERWISE SPECIFIED

\begin{tabular}{|l|r|}
\hline Simulation parameter & Value \\
\hline \hline Subcarrier block bandwidth, $W$ [Hertz] & $180 \mathrm{k}$ \\
\hline Number of RNs per macrocell, $M$ & $\{0,1,2,3\}$ \\
\hline Number of subcarriers blocks, $N$ & 12 \\
\hline Number of UEs, $K$ & $(4,4,4)$ \\
\hline Antenna configuration, $\left(N_{B}, N_{R}, N_{U}\right)$ & 0.1 \\
\hline Semi-orthogonality parameter, $\alpha$ & $\{1.5,2.5,3.5,4.5\}$ \\
\hline Inter-site distance (ISD), [km] & 1 and 2 \\
\hline $\begin{array}{l}\text { Minimum number of receive dimensions } \\
\text { at the RNs and UEs, } S^{R} \text { and } S^{U}\end{array}$ & 0.7 \\
\hline $\begin{array}{l}\text { Ratio of BS-to-RN distance to the cell } \\
\text { radius, } D_{r}\end{array}$ & 0 \\
\hline SNR gap of wireless transceivers, $\Delta \gamma[\mathrm{dB}]$ & $\{0,12,24$, \\
\hline $\begin{array}{l}\text { Maximum total transmission power of the } \\
\text { BS and RNs, } P_{m a x}^{B} \text { and } P_{\text {max }}^{R}[\mathrm{dBm}]\end{array}$ & $36,48,60\}$ \\
\hline $\begin{array}{l}\text { Fixed power rating of the BS, } \\
P_{C}^{B} \text { [Watts] [42], [48] }\end{array}$ & $32.306 N_{B}$ \\
\hline $\begin{array}{l}\text { Fixed power rating of RNs, } \\
P_{C}^{R} \text { [Watts] [42], [48] }\end{array}$ & $21.874 N_{R}$ \\
\hline $\begin{array}{l}\text { Reciprocal of the BS power amplifier's } \\
\text { drain efficiency, } \xi^{B} \text { [42], [48] }\end{array}$ & $3.24 N_{B}$ \\
\hline $\begin{array}{l}\text { Reciprocal of the RNs' power amplifier's } \\
\text { drain efficiency, } \xi^{R} \text { [42], [48] }\end{array}$ & $4.04 N_{R}$ \\
\hline Noise power spectral density, $N_{0}[\mathrm{dBm} / \mathrm{Hz}]$ & -174 \\
\hline $\begin{array}{l}\text { Convergence threshold, } \epsilon \\
\text { Number of channel samples }\end{array}$ & $10^{-8}$ \\
\hline
\end{tabular}

loss model of [49] and assumed that all BS-UE and RN-UE links are NLOS links, since they are typically blocked by buildings and other large obstructing objects, while all BS-RN links may realistically be assumed to be line-of-sight links, since the RNs may be strategically positioned on tall buildings to create strong wireless backhaul links. Furthermore, independently and randomly generated set of UE locations as well as fading channel realizations were used. Again, for benchmarking we employ a baseline algorithm, which relies on random SMC selections and equal power allocation (EPA) across the selected SMCs. This algorithm is termed as the EPA algorithm.

The attainable performance of both the full-IA and partial-IA protocols is explored and these results are obtained by employing the optimized power control variables and group selection variables in the actual system model. Therefore, the results reflect the actual ESE achieved rather than the optimized OF value of (60), which is optimistic, since it does not account for any potential OCI remaining after employing the partial-IA protocol.

\section{A. The Variation of ASE and ESE for Different Values of $P_{\max }^{B}$ and $P_{\max }^{R}$}

The effects of varying both $P_{\max }^{B}$ and $P_{\max }^{R}$ are demonstrated in Fig. 3. Observe that the partial-IA protocol outperforms the full-IA protocol for all the power constraints considered. This is due to the requirements of (8), (9), (20) and (24), which restrict the number of data streams that the BSs can transmit simultaneously in each phase. The full-IA protocol imposes more restrictive constraints than the partial-IA protocol, since the partial-IA protocol only requires that the Rx BFMs has to eliminate the ICI,

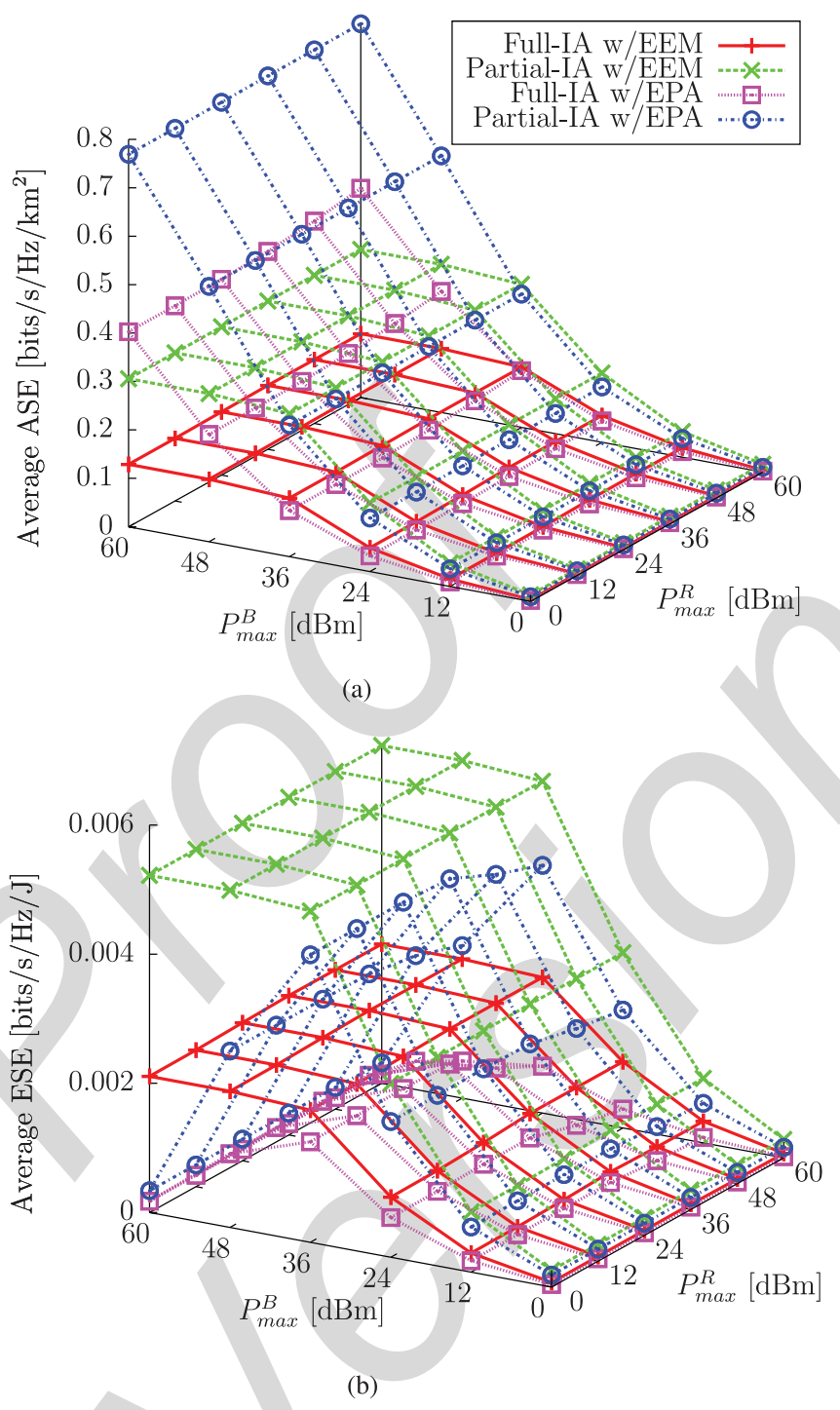

Fig. 3. The average achievable ASE and ESE when using the ESEM and EPA algorithms with either full-IA or partial-IA, for varying $P_{\max }^{B}$ and $P_{\max }^{R}$, and using the parameters in Table II with $M=2$ and an ISD of $1.5 \mathrm{~km}$. (a) Surface plots of the achievable ASE when using the ESEM and EPA algorithms. (b) Surface plots of the achievable ESE when using the ESEM and EPA algorithms.

rather than both the ICI and OCI that the full-IA protocol has to null. Observe furthermore that the EPA algorithms achieve higher ASE values than their ESEM algorithmic counterparts at high $P_{\max }^{B}$ values. However, this is achieved at a higher cost to the ESE obtained from using the EPA algorithms, when compared to their ESEM counterparts. In fact, in the low to medium $P_{\max }^{B}$ regime, both the SEM and ESEM correspond to the same solution, as demonstrated in our previous works of [25]-[27]. This results in a higher ASE for the ESEM algorithm than for the heuristic EPA algorithm. As the value of $P_{\max }^{B}$ increases, the EPA continues to allocate more power, which increases the ASE obtained, without any cognizance to the ESE performance.

However, the ASE and ESE obtained does not increase significantly upon increasing $P_{\max }^{R}$. This can be attributed to the low multiplexing gain specified in these experiments, given that $S^{R}=1$. The results of the next subsection explore the effects of varying the requirements imposed on $S^{U}$ and $S^{R}$. 


\section{B. The Variation of ASE and ESE for Different Values of $S^{U}$ and $S^{R}$}

Fig. 4 shows the results obtained upon varying $S^{U}$ and $S^{R}$. Once again, the partial-IA protocol outperforms the full-IA protocol in terms of both its ASE and ESE performances. Additionally, we observe that the EPA algorithm performs worse than the ESEM algorithm for all cases. Increasing $S^{U}$ has a marginal effect on the ASE and ESE obtained for both protocols. However, increasing $S^{R}$ does lead to an increase in SE, when employing the partial-IA protocol, albeit at a cost to ESE resulting from the fixed power dissipation costs of the RNs. Observe that increasing $S^{R}$ reduces the ASE attained when using the full-IA protocol. This may be explained by the detrimental effects of the constraints imposed on the multiplexing gain of the BSs' transmissions when employing the full-IA protocol, because increasing $S^{R}$ imposes a substantial reduction on both (8) and (20), when multiple RNs are operated in each macrocell. This reduction in ASE is not so dominant for the partial-IA protocol, since the increase in the multiplexing gain of the RNs' transmissions outweighs the detrimental effects of imposing a multiplexing gain restriction at the BSs due to (24). Additionally, the potential multiplexing gain attained at the BSs in the first transmission phase, given by (9), is not affected by the increase of $S^{R}$.

\section{The Variation of ASE and ESE for Different Values of $M$ and Inter-Site Distance}

As shown in Fig. 5, both the achievable ASE and ESE decreases as the ISD is increased, indicating that the effect of a higher path-loss on the channel gains has a more grave detrimental effect on both the ASE and ESE than the beneficial effects of the reduced interference levels. Once again, the EPA algorithm performs worse than their ESEM algorithmic counterparts. Additionally, the ASE attained, when using the full-IA protocol is slightly reduced upon increasing $M$ due to both (8) and (20), while the ESE achieved is reduced, as the power dissipation of the system is increased upon increasing $M$. Furthermore, the ASE obtained when using the partial-IA protocol peaks for $M=1$, but decreases slightly, upon increasing $M$ further, since then the multiplexing gain experienced during the second phase is reduced as indicated by (24). By contrast, the ESEM of the partial-IA protocol only decreases upon increasing $M^{10}$.

\section{CONCLUSION}

In this paper, a multi-user, multi-relay, multi-cell MIMO system model is studied. In order to avoid the excessive interference inflicted by the multiple transmission sources, a pair of distributed IA protocols were designed. The first, termed as full-IA, completely avoids any interference by finding RxBFMs, which entirely eliminate the interference imposed at the receivers. However, this comes at a cost to the spatial multiplexing gain of the BSs, which limits the number of

\footnotetext{
${ }^{10}$ In fact, when $M=0$ or $S^{R}=0$ we arrive at a special case of the partial-IA protocol, which is similar to the conventional single cell multi-user ZFBF in the absence of RNs. However, the proposed partial-IA protocol represents a sophisticated extension of classic ZFBF to the broad class of multi-relay aided multi-cell networks, which have been combined with intelligent user selection.
}
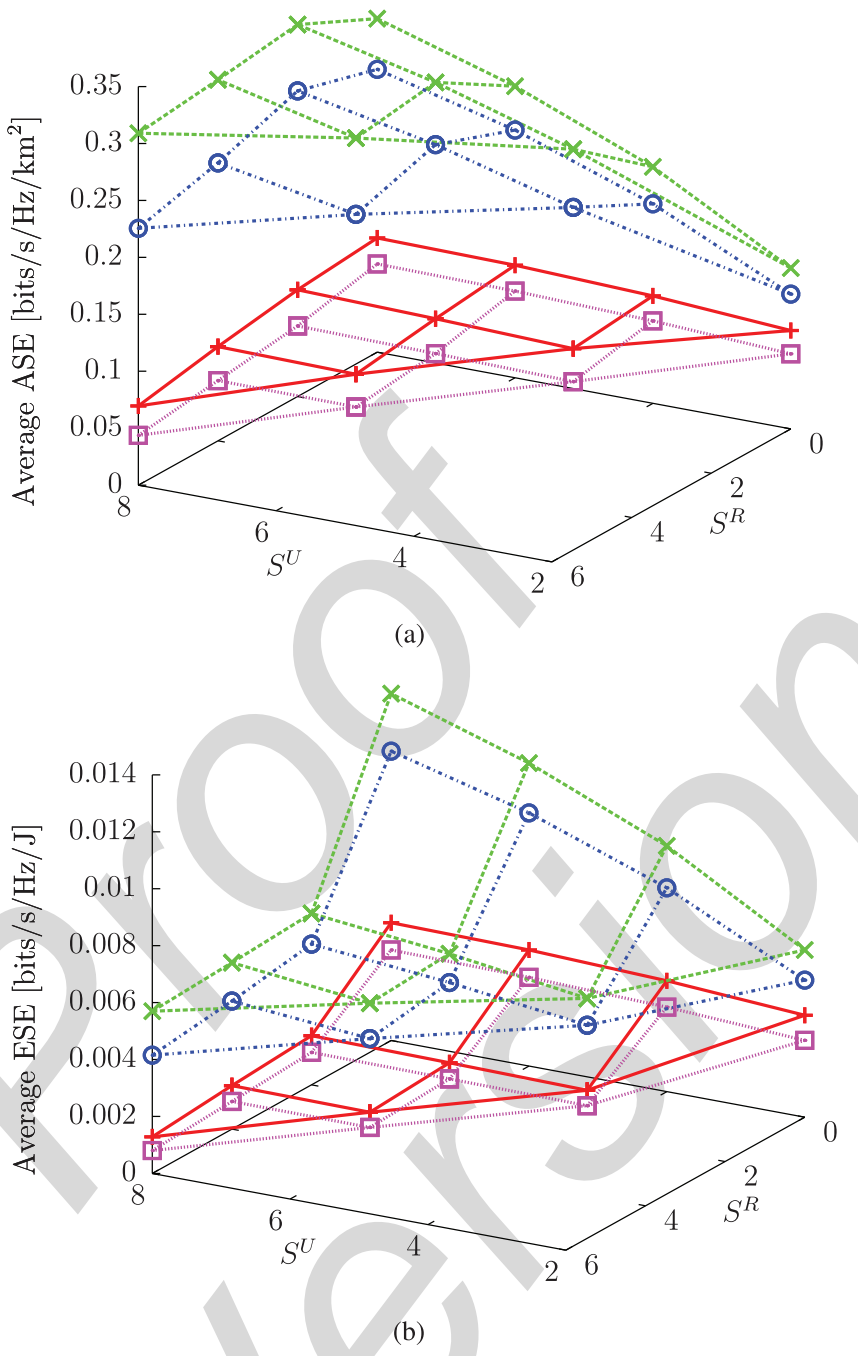

Fig. 4. The average achievable ASE and ESE when using the ESEM and EPA algorithms with either full-IA or partial-IA, for varying $S^{U}$ and $S^{R}$, and using the parameters in Table II with $M=2, P_{\max }^{B}=30 \mathrm{dBm}, P_{\max }^{R}=20 \mathrm{dBm}$ and an ISD of $1.5 \mathrm{~km}$. The legend is as presented in Fig. 3. (a) Surface plots of the achievable ASE when using the ESEM and EPA algorithms. (b) Surface plots of the achievable ESE when using the ESEM and EPA algorithms.

DL transmission streams. The second transmission protocol, namely partial-IA, aims for striking a balance between the spatial multiplexing gain and interference contamination by finding RxBFMs, which only null the interference emerging from sources within the same macrocell. Employing the RxBFMs created by either of these transmission protocols results in a list of SMCs, which correspond to data streams that may be conveyed by the BS. We formally defined the problem of maximizing the ESE by optimally choosing the SMCs as well as by appropriately choosing their power control variables. The resultant non-convex optimization problem was converted into a convex optimization problem with the aid of carefully chosen variable relaxations and transformations, which was then solved using the classic dual decomposition and subgradient methods [37], that may be implemented distributively at each BS. We characterized the attainable ASE and ESE performances of both protocols for a range of system parameters, while comparing the performance of our ESEM algorithm to that of a baseline EPA algorithm. To summarize, 


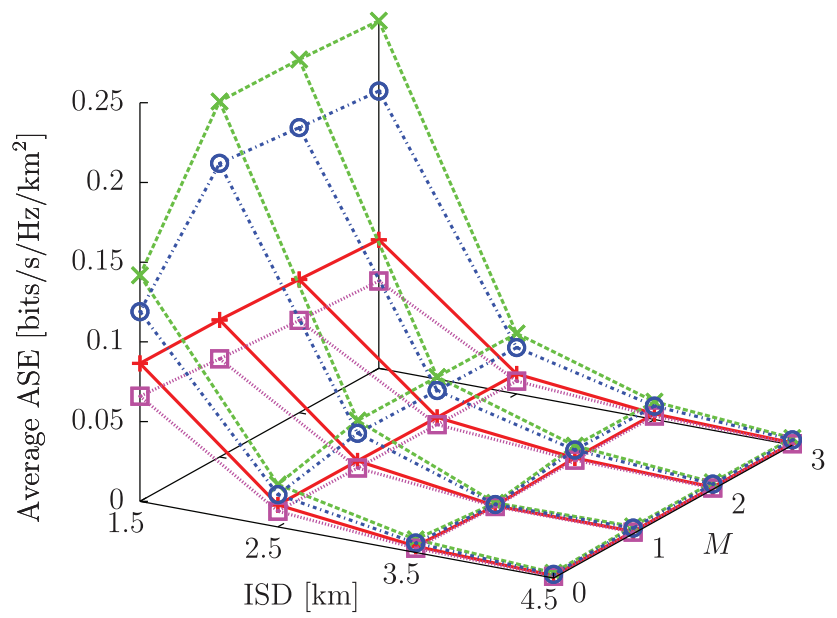

(a)

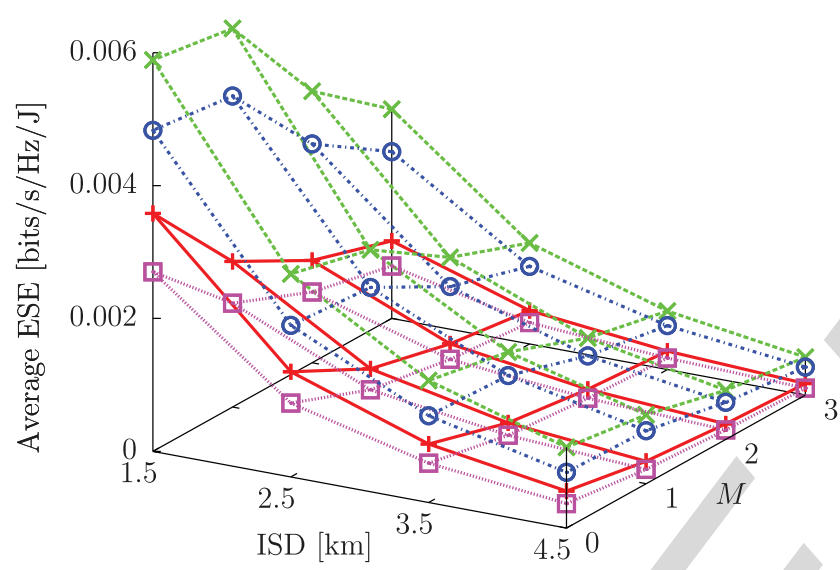

(b)

Fig. 5. The average achievable ASE and ESE when using the ESEM and EPA algorithms with either full-IA or partial-IA, for varying $M$ and ISD, and using the parameters in Table II with $P_{\max }^{B}=30 \mathrm{dBm}, P_{\max }^{R}=20 \mathrm{dBm}$. The legend is as presented in Fig. 3. (a) Surface plots of the achievable ASE when using the ESEM and EPA algorithms. (b) Surface plots of the achievable ESE when using the ESEM and EPA algorithms.

the ESEM algorithm outperforms the EPA algorithm in terms of ESE, while surprisingly the partial-IA protocol outperforms the full-IA protocol in all cases. For the cell sizes considered, the path-loss mitigates the majority of the OCI, and thus the full-IA protocol actually over-compensates, when reducing the number of available transmit dimensions at the transmitters to facilitate IA.

\section{REFERENCES}

[1] D. Lopez-Perez, A. Valcarce, G. de la Roche, and J. Zhang, "OFDMA femtocells: A roadmap on interference avoidance," IEEE Commun. Mag., vol. 47, no. 9, pp. 41-48, Sep. 2009.

[2] P. Bhat, S. Nagata, L. Campoy, I. Berberana, T. Derham, G. Liu, X. Shen, P. Zong, and J. Yang, "LTE-advanced: An operator perspective," IEEE Commun. Mag., vol. 50, no. 2, pp. 104-114, Feb. 2012.

[3] O. El Ayach, S. Peters, and R. Heath, Jr., "The practical challenges of interference alignment," IEEE Wireless Commun. Mag., vol. 20, no. 1, pp. 35-42, Feb. 2013.

[4] C. Han, T. Harrold, S. Armour, I. Krikidis, S. Videv, P. Grant, H. Haas, J. Thompson, I. Ku, C.-X. Wang, T. A. Le, M. Nakhai, J. Zhang, and L. Hanzo, "Green radio: Radio techniques to enable energy-efficient wireless networks," IEEE Commun. Mag., vol. 49, no. 6, pp. 46-54, Jun. 2011.
[5] J. Laneman, D. Tse, and G. Wornell, "Cooperative diversity in wireless networks: Efficient protocols and outage behavior," IEEE Trans. Inf. Theory, vol. 50, no. 12, pp. 3062-3080, Dec. 2004.

[6] M. Maddah-Ali, A. Motahari, and A. Khandani, "Communication over X channel: Signalling and multiplexing gain," Univ. Waterloo, Canada, Tech.Rep. UW-ECE-2006-12, Jul. 2006.

[7] M. Maddah-Ali, A. Motahari, and A. Khandani, "Communication over X channel: Signaling and performance analysis," Univ. Waterloo, Canada, Tech. Rep. UW-ECE-2006-27, Dec. 2006.

[8] M. Maddah-Ali, A. Motahari, and A. Khandani, "Signaling over MIMO multi-base systems: Combination of multi-access and broadcast schemes," in Proc. IEEE Int. Symp. Inf. Theory, Seattle, WA, USA, Jul. 2006, pp. 2104-2108.

[9] M. Maddah-Ali, A. Motahari, and A. Khandani, "Communication over MIMO X channels: Interference alignment, decomposition, and performance analysis," IEEE Trans. Inf. Theory, vol. 54, no. 8, pp. 3457-3470, Aug. 2008.

[10] V. Cadambe and S. Jafar, "Interference alignment and degrees of freedom of the $K$-user interference channel," IEEE Trans. Inf. Theory, vol. 54 , no. 8, pp. 3425-3441, Aug. 2008.

[11] C. Suh and D. Tse, "Interference alignment for cellular networks," in Proc. Allerton Conf. Commun., Contr., Comput., Urbana-Champaign, IL, USA, Sep. 2008, pp. 1037-1044.

[12] H. Gao, T. Lv, D. Fang, S. Yang, and C. Yuen, "Limited feedback-based interference alignment for interfering multi-access channels," IEEE Commun. Lett., vol. 18, no. 4, pp. 540-543, Apr. 2014.

[13] C. Suh, M. Ho, and D. Tse, "Downlink interference alignment," IEEE Trans. Commun., vol. 59, no. 9, pp. 2616-2626, Sep. 2011.

[14] D. Kim and M. Torlak, "Optimization of interference alignment beamforming vectors," IEEE J. Sel. Areas Commun., vol. 28, no. 9, pp. 1425-1434, Dec. 2010.

[15] B. Da and R. Zhang, "Exploiting interference alignment in multi-cell cooperative OFDMA resource allocation," in Proc. IEEE Global Telecommun. Conf., Houston, TX, USA, Dec. 2011, pp. 1-5.

[16] K. Gomadam, V. Cadambe, and S. Jafar, "A distributed numerical approach to interference alignment and applications to wireless interference networks," IEEE Trans. Inf. Theory, vol. 57, no. 6, pp. 3309-3322, Jun. 2011.

[17] M. Rezaee and S. Nader-Esfahani, "Interference alignment for downlink transmission of multiple interfering cells," IEEE Wireless Commun. Lett., vol. 1, no. 5, pp. 460-463, Oct. 2012.

[18] J. Tang and S. Lambotharan, "Interference alignment techniques for MIMO multi-cell interfering broadcast channels," IEEE Trans. Commun., vol. 61, no. 1, pp. 164-175, Feb. 2013.

[19] H. J. Yang, W.-Y. Shin, B. C. Jung, C. Suh, and A. Paulraj, "Opportunistic downlink interference alignment," [Online]. Available: http:// arxiv.org/abs/1312.7198

[20] T. Yoo and A. Goldsmith, "On the optimality of multiantenna broadcast scheduling using zero-forcing beamforming," IEEE J. Sel. Areas Commun., vol. 24, no. 3, pp. 528-541, Mar. 2006.

[21] A. Goldsmith, Wireless Communications. New York, NY, USA: Cambridge Univ. Press, 2005.

[22] C. Xiong, G. Li, S. Zhang, Y. Chen, and S. Xu, "Energy-efficient resource allocation in OFDMA networks," IEEE Trans. Commun., vol. 60, no. 12 , pp. 3767-3778, Dec. 2012.

[23] D. Ng, E. Lo, and R. Schober, "Energy-efficient resource allocation in multi-cell OFDMA systems with limited backhaul capacity," IEEE Trans. Wireless Commun., vol. 11, no. 10, pp. 3618-3631, Oct. 2012.

[24] R. Devarajan, S. Jha, U. Phuyal, and V. Bhargava, "Energy-aware resource allocation for cooperative cellular network using multi-objective optimization approach," IEEE Trans. Wireless Commun., vol. 11, no. 5, pp. 1797-1807, May 2012.

[25] K. T. K. Cheung, S. Yang, and L. Hanzo, "Achieving maximum energy-efficiency in multi-relay OFDMA cellular networks: A fractional programming approach," IEEE Trans. Commun., vol. 61, no. 7, pp. 2746-2757, Jul. 2013.

[26] K. T. K. Cheung, S. Yang, and L. Hanzo, "Maximizing energy-efficiency in multi-relay OFDMA cellular networks," in Proc. IEEE Global Telecommun. Conf., Atlanta, GA, USA, Dec. 2013, pp. 2767-2772.

[27] K. T. K. Cheung, S. Yang, and L. Hanzo, "Spectral and energy spectral efficiency optimization of joint transmit and receive beamforming based multi-relay MIMO-OFDMA cellular networks," IEEE Trans. Wireless Commun., vol. 13, no. 11, pp. 6147-6165, Nov. 2014.

[28] D. Gesbert, S. Hanly, H. Huang, S. S. Shitz, O. Simeone, and W. Yu, "Multi-cell MIMO cooperative networks: A new look at interference," IEEE J. Sel. Areas Commun., vol. 28, no. 9, pp. 1380-1408, Dec. 2010.

[29] W. Dinkelbach, "On nonlinear fractional programming," Manage. Sci., vol. 13, pp. 492-498, Mar. 1967.

[30] M. Avriel, W. E. Diewert, S. Schaible, and I. Zang, Generalized Concavity. New York, NY, USA: Plenum, 1988. 
[31] S. Boyd and L. Vandenberghe, Convex Optimization. New York, NY, USA: Cambridge Univ. Press, 2004.

[32] C. Isheden, Z. Chong, E. Jorswieck, and G. Fettweis, "Framework for link-level energy efficiency optimization with informed transmitter," IEEE Trans. Wireless Commun., vol. 11, no. 8, pp. 2946-2957, Aug. 2012.

[33] H. Sung, S.-H. Park, K.-J. Lee, and I. Lee, "Linear precoder designs for $K$-user interference channels," IEEE Trans. Wireless Commun., vol. 9 , no. 1, pp. 291-301, Jan. 2010.

[34] G. Alexandropoulos and C. Papadias, "A reconfigurable distributed algorithm for $K$-user MIMO interference networks," in Proc. IEEE Int. Conf. Commun., Budapest, Hungary, Jun. 2013, pp. 3063-3067.

[35] K. Ronasi, B. Niu, V. W. Wong, S. Gopalakrishnan, and R. Schober, "Throughput-efficient scheduling and interference alignment for MIMO wireless systems," IEEE Trans. Wireless Commun., vol. 13, no. 4, pp. 1779-1789, Apr. 2014.

[36] X. Chen and C. Yuen, "Performance analysis and optimization for interference alignment over MIMO interference channels with limited feedback," IEEE Trans. Signal Process., vol. 62, no. 7, pp. 1785-1795, Apr. 2014.

[37] D. Palomar and M. Chiang, "A tutorial on decomposition methods for network utility maximization," IEEE J. Sel. Areas Commun., vol. 24, no. 8, pp. 1439-1451, Aug. 2006.

[38] G. Raleigh and J. Cioffi, "Spatio-temporal coding for wireless communication," IEEE Trans. Commun., vol. 46, no. 3, pp. 357-366, Mar. 1998.

[39] N. Ul Hassan and M. Assaad, "Low complexity margin adaptive resource allocation in downlink MIMO-OFDMA system," IEEE Trans. Wireless Commun., vol. 8, no. 7, pp. 3365-3371, Jul. 2009.

[40] R. Blum, "MIMO capacity with interference," IEEE J. Sel. Areas Commun., vol. 21, no. 5, pp. 793-801, Jun. 2003.

[41] L. Hanzo, O. Alamri, M. El-Hajjar, and N. Wu, Near-Capacity MultiFunctional MIMO Systems: Sphere-Packing, Iterative Detection and Cooperation. New York, NY, USA: Wiley-IEEE, 2009.

[42] G. Auer, O. Blume, V. Giannini, I. Godor, M. A. Imran, and Y. Jading et al., "D2.3: Energy efficiency analysis of the reference systems, areas of improvements and target breakdown," FP7 INFSO-ICT-247733 EARTH (Energy Aware Radio and NeTwork TecHnologies), Nov. 2010 [Online]. Available: www.ict-earth.eu/publications/deliverables/deliverables.html

[43] D. P. Bertsekas, Nonlinear Programming. Belmont, MA, USA: Athena Scientific, 1999.

[44] W. Yu and R. Lui, "Dual methods for nonconvex spectrum optimization of multicarrier systems," IEEE Trans. Commun., vol. 54, no. 7, pp. 1310-1322, July 2006.

[45] D. Ng, E. Lo, and R. Schober, "Dynamic resource allocation in MIMOOFDMA systems with full-duplex and hybrid relaying," IEEE Trans. Commun., vol. 60, no. 5, pp. 1291-1304, May 2012.

[46] D. Ng, E. Lo, and R. Schober, "Energy-efficient resource allocation for secure OFDMA systems," IEEE Trans. Veh. Technol., vol. 61, no. 6, pp. 2572-2585, Jul. 2012.

[47] K. Seong, M. Mohseni, and J. Cioffi, "Optimal resource allocation for OFDMA downlink systems," in Proc. IEEE Int. Symp. Inf. Theory, Seattle, WA, USA, Jul. 2006, pp. 1394-1398.

[48] O. Arnold, F. Richter, G. Fettweis, and O. Blume, "Power consumption modeling of different base station types in heterogeneous cellular networks," in Proc. Future Netw. Mobile Summit, Florence, Italy, Jun. 2010.

[49] Further advancements for E-UTRA, physical layer aspects (Release 9), TR 36.814 V9.0.0, 3GPP, Mar. 2010.

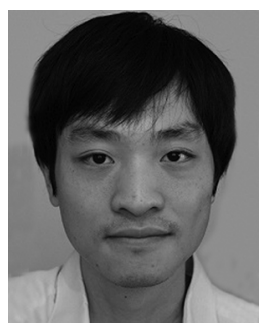

Kent Tsz Kan Cheung (S'09) received the B.Eng. degree (first class honors) in electronic engineering from the University of Southampton, Southampton, U.K., in 2009. In 2015, he received the Ph.D. degree in wireless communications at the same institution and is now a postdoctoral researcher there.

His research interests include energy-efficiency, multicarrier MIMO communications, cooperative communications, resource allocation, and optimization.

Dr. Cheung was a recipient of the EPSRC Industrial CASE award in 2009, and was involved with the Core 5 Green Radio project of the Virtual Centre of Excellence in Mobile and Personal Communications (Mobile VCE).

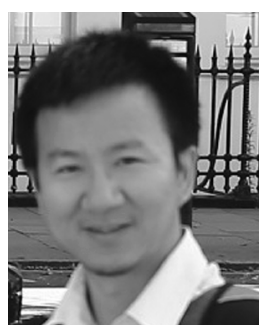

Shaoshi Yang (S'09-M'13) received the B.Eng. degree in information engineering from Beijing University of Posts and Telecommunications (BUPT), Beijing, China, in July 2006, the Ph.D. degree in electronics and electrical engineering from the University of Southampton, United Kingdom, in December 2013, and the Ph.D. degree in signal and information processing from BUPT in March 2014.

He is now working as a Postdoctoral Research Fellow with the University of Southampton, U.K. From November 2008 to February 2009, he was an Intern Research Fellow with the Communications Technology Lab (CTL), Intel Labs, Beijing, where he focused on Channel Quality Indicator Channel $(\mathrm{CQICH})$ design for mobile WiMAX $(802.16 \mathrm{~m})$ standard. His research interests include MIMO signal processing, green radio, heterogeneous networks, cross-layer interference management, convex optimization, and its applications. He has published more than 30 research papers in IEEE journals and conferences.

Dr. Yang has received a number of academic and research awards, including the prestigious Dean's Award for Early Career Research Excellence at University of Southampton, the PMC-Sierra Telecommunications Technology Paper Award at BUPT, the Electronics and Computer Science (ECS) Scholarship of University of Southampton, and the Best Ph.D. Thesis Award of BUPT. He is a member of the IEEE/IET, and a junior member of Isaac Newton Institute for Mathematical Sciences, Cambridge University, U.K. He also serves as a Technical Program Chair of several major IEEE conferences, including IEEE ICC, GLOBECOM, PIMRC, ICCVE, HPCC, and as a Guest Associate Editor of IEEE JOURNAL ON SELECTED AREAS IN COMMUNICATIONS (https://sites. google.com/site/shaoshiyang/).

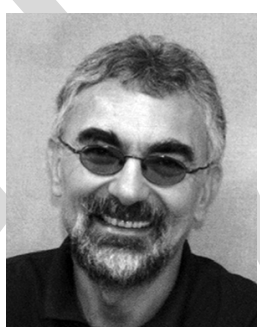

Lajos Hanzo (F'08) received the D.Sc. degree in electronics in 1976 and the doctorate degree in 1983 from the Technical University of Budapest. In 2009, he was awarded the honorary doctorate "Doctor Honoris Causa" by the Technical University of Budapest.

During his 35-year career in telecommunications he has held various research and academic posts in Hungary, Germany, and the United Kingdom. Since 1986, he has been with the School of Electronics and Computer Science, University of Southampton, U.K., where he holds the Chair in Telecommunications. He has successfully supervised $80 \mathrm{Ph}$.D. students, coauthored 20 John Wiley/IEEE Press books on mobile radio communications totalling in excess of 10000 pages, published more than 1500 research entries at IEEE Xplore. Currently he is directing a 60 -strong academic research team, working on a range of research projects in the field of wireless multimedia communications sponsored by industry, the Engineering and Physical Sciences Research Council (EPSRC) UK, the European IST Programme and the Mobile Virtual Centre of Excellence (VCE), UK.

Dr. Hanzo acted both as Technical Program Chairperson and General Chair of IEEE conferences, presented keynote lectures, and has been awarded a number of distinctions. He is a Fellow of EURASIP. He is also a Governor of the IEEE VTS. During 2008-2012, he was the Editor-in-Chief of the IEEE Press and a Chaired Professor at Tsinghua University, Beijing. His research was funded by the European Research Council's Senior Research Fellow Grant. For further information on research in progress and associated publications please refer to http://www-mobile.ecs.soton.ac.uk. 